\title{
VII. Der Rollentausch
}

Nach der Ratifizierung begannen auf beiden Seiten die Vorbereitungen zur Umsetzung des Abkommens. Ein Verwaltungsapparat für die Bestellung und Bezahlung der Güter, für den Transport nach Israel und die Verteilung im Land mußte geschaffen werden. Einige Aufgaben erforderten eine enge Zusammenarbeit zwischen beiden Seiten, andere ließen sich unabhängig durchführen. Das Schilumimabkommen enthält allgemeine Richtlinien über die Beschaffenheit der ausführenden Institutionen sowie über das Kauf-, Kontroll-, Zahlungs- und Lieferverfahren.

Die israelische Regierung war verpflichtet, in der Bundesrepublik eine Einkaufsdelegation des Staates Israel, die sogenannte Israel-Mission, zu eröffnen. ${ }^{1}$ Die Bundesrepublik trug die Verantwortung für die Gründung eines gemischten Ausschusses, zusammengesetzt aus Vertretern beider Regierungen. Als Sitz der Israel-Mission wurde Köln vereinbart. Die Kölner Stadtverwaltung stellte ein Grundstück zur Verfügung, auf dem später das Gebäude der Mission errichtet wurde. Der gemischte Ausschuß residierte in Frankfurt am Main, gleichzeitig auch Sitz der Bundesstelle für den Warenverkehr der gewerblichen Wirtschaft, die für die Durchführung des Abkommens auf deutscher Seite verantwortlich war.

Köln liegt auf halbem Weg zwischen Düsseldorf, dem Zentrum der Schwerindustrie, und der damaligen Bundeshauptstadt Bonn. Um den Eindruck einer offiziellen diplomatischen Vertretung zu vermeiden, die als formelle Anerkennung der Bundesrepublik durch Israel interpretiert worden wäre, bestand die israelische Regierung darauf, die Mission nicht in Bonn anzusiedeln. Jerusalem war zu jenem Zeitpunkt streng darauf bedacht, jeglichen Anschein zu vermeiden, als unterhalte man diplomatische Beziehungen zur Bundesrepublik. Köln galt deshalb als idealer Kompromiß. Die Israelis waren zudem - vermutlich aus praktischen Gründen daran interessiert, den Sitz des gemischten Ausschusses in nächster Nachbarschaft anzusiedeln. Da sich die Bundesstelle für Warenverkehr jedoch in Frankfurt am Main befand, lehnte Bonn diesen Wunsch ab. ${ }^{2}$

\section{Die israelische Schilumimexekutive}

Die ausführenden Organe auf israelischer Seite setzten sich aus zwei Teilen zusammen: aus der Einkaufsmission in Köln und zwei provisorischen Zweigstellen in West-Berlin und Hamburg sowie aus der Schilumimgesellschaft mit Sitz in Tel Aviv und einer Zweigstelle in der nordisraelischen Hafenstadt Haifa. Die oberste Entscheidungsgewalt lag bei den staatlichen Kontrollorganen in Jerusalem.

1 Documents Relating, Artikel 12.

2 Max Adenauer an Konrad Adenauer vom 6. 12. 1952, PA, 244-13 II 17178/52. 
Die israelische Einkaufsmission war zwar im Schilumimabkommen verankert, doch außer der Gewährung diplomatischer Privilegien und Immunitäten wurde ihr Aufbau und ihre personelle Zusammensetzung weitgehend im dunkeln gelassen. ${ }^{3}$ Am 4. Mai 1953 nahm die Mission ihre Arbeit auf, bei gleichzeitiger Schließung des Konsulats in München. Der Leiter der Israel-Mission wurde in das diplomatische Korps aufgenommen und gemäß diplomatischem Protokoll regelmäßig zu offiziellen Anlässen eingeladen. Da der Staat Israel die Bundesrepublik de jure nicht anerkannte, hatte die Mission keinen rechtlichen Anspruch auf diplomatische Privilegien. Die gewährten Vorrechte waren somit als Geste des guten Willens zu betrachten. Ein solches Privileg war etwa das Recht auf Führung einer Konsularabteilung, die sich mit der Ausstellung von Sichtvermerken und anderen konsularischen Diensten beschäftigte. Obwohl die Eröffnung dieser Abteilung durchaus im Interesse der Bundesrepublik lag, waren dazu langwierige Verhandlungen erforderlich. Im bilateralen Dokument wurde ausdrücklich festgehalten, $\mathrm{da} ß$ die Konsularabteilung nicht als Ausdruck von diplomatischen Beziehungen zu werten sei. ${ }^{4} \mathrm{Da}$ die Israel-Mission formal dem Finanzminister unterstellt war, galten die Vertreter des Außenministeriums dort als Außenseiter. Dies provozierte im israelischen Außenministerium und in anderen israelischen Regierungseinrichtungen den Vorwurf, die Architekten der israelischen Außenpolitik seien nicht oder zumindest nicht entscheidend an der Gestaltung der Aktivitäten der Mission beteiligt. ${ }^{5}$

Beide Regierungen widmeten dem Status der israelischen Einkaufsmission reifliche Überlegungen. Die Israelis studierten das Beispiel der sowjetischen Handelsmission in Deutschland zwischen den beiden Weltkriegen, die die wirtschaftlichen und politischen Interessen Moskaus ohne offiziellen diplomatischen Status vertreten hatte. ${ }^{6}$ Finnland war aus politischen Gründen in Bonn auch nur durch eine Handelsmission vertreten. Dennoch nahm diese Vertretung ebenfalls politische bzw. diplomatische Aufgaben wahr - also ein weiteres mögliches Modell für die israelische Mission. Experten für internationales Recht auf deutscher Seite stellten ähnliche Untersuchungen über historische Präzedenzfälle an, waren aber in erster Linie daran interessiert, Näheres über die israelischen Vorstellungen zur IsraelMission zu erfahren. ${ }^{7}$ Schließlich gewährte die Bundesregierung der Mission großzügige Privilegien - wohl im Hinblick auf die Förderung der bilateralen Beziehungen.

Neben einer erweiterten Handelsabteilung, dem Kern der Einrichtung, beherbergte die israelische Einkaufsmission eine Rechtsabteilung und eine Abteilung

3 Documents Relating, Artikel 12, Paragraph (f), S. 131-133.

4 Memo von Nostiz vom 2. 8. 1953, Memo von Reismann vom 29. 1. 1954, PA, 210-03/ 92.19, 82-03/35; Yachil, Köln, an das israelische Außenministerium, Konsularabteilung, an den Rechtsberater und die Abteilung Westeuropa vom 11.3. 1954, ISA, 617/16.

5 Meroz, In schwieriger Mission, Kap. 1; Interview mit Nachum Shamir am 20.5. und 17. 6. 1987; Yachil an Eytan vom 2. 2. 1954; Zeev Scheck, Politischer Sekretär des Außenministers, an Sharett vom 15. 2. 1954, ISA, 2418/14.

6 G. Viemanas an Maurice Fischer vom 9. 7. 1952 ISA, 155/1.

7 Niederschrift über die 10. Sitzung des Rechtsausschusses vom 18. 7. 1952, BArch, B 102/ 7019. 
für Öffentlichkeitsarbeit, die für die regelmäßige Herausgabe des Bulletins Israel Informationsdienst verantwortlich war. Die Handelsabteilung gliederte sich in diverse Unterabteilungen je nach Importgut: Eisen und Stahl, Maschinen, Chemikalien, Holz etc. Weitere Unterabteilungen befaßten sich mit Transport, Versicherung, Bankwesen, Bankverkehr mit deutschen, holländischen und amerikanischen Banken, Finanzen und Rechnungsprüfung. Das Büro der Mission beschäftigte zahlreiche israelische und deutsche Schreibkräfte, und die Kontrolle über die Einrichtung wurde von einer Sonderabteilung wahrgenommen, der auch ein Vertreter des israelischen Rechnungshofes angehörte. Die Stellung des politischen Beirats, faktisch der Vertreter des Außenministeriums, gab zu einigen internen Diskussionen Anlaß. Mindestens in zwei Fällen gelang es dem Leiter der Mission, Felix Eliezer Shinnar, den Beirat seines Amtes zu entheben, nämlich Chaim Yachil in den frühen und Yochanan Meroz in den späten fünfziger Jahren. Nur Leo Savir harrte in den sechziger Jahren länger auf diesem Posten aus, möglicherweise wegen Shinnars häufiger Abwesenheit in jenen Jahren. Die personell bescheiden ausgestattete Konsularabteilung unterstand dem Außenministerium. 1955 trat Oberst Avigdor Ben-Gal als inoffizieller Vertreter des israelischen Verteidigungsministeriums in die Mission ein. Nach Ben-Gals Tod wurde diese Funktion Mitte der sechziger Jahre sodann von Oberst Asher Arbel übernommen. Arbel wurde oft mit dem israelischen Geheimdienst (Mossad) in Verbindung gebracht und war möglichweise dessen Vertreter in der Bundesrepublik. Gelegentlich führte er geheime Missionen im Auftrag seiner Vorgesetzten und des israelischen Verteidigungsministeriums durch. Arbel pflegte enge Kontakte zu lokalen Polizeistellen in Köln. ${ }^{8}$

Am Anfang gehörten der vorübergehend in den Räumlichkeiten der jüdischen Religionsgemeinde in Köln untergebrachten Mission rund dreißig Experten, darunter auch amerikanische und britische Fachleute jüdischer Herkunft, an. Später wurden die "Ausländer" schrittweise von Israelis abgelöst. ${ }^{9}$ Der Leiter der Handelsabteilung amtierte gleichzeitig als Stellvertreter von Missionsleiter Shinnar. Auf dem Höhepunkt ihrer Tätigkeit in den späten fünfziger Jahren beschäftigte die Israel-Mission laut Bericht vom 1. März 1959158 Mitarbeiter. $\mathrm{Zu}$ diesem Zeitpunkt setzte sich das Personal nur noch aus israelischen und westdeutschen Staatsbürgern zusammen. ${ }^{10}$ Am 1. April 1963 wurde die Belegschaft dann auf 13 Mitarbeiter reduziert. ${ }^{11}$ Nach der Erfüllung ihres Auftrags im Mai 1965 und der Eröffnung der neuen Botschaft in Bad Godesberg übergab die Mission das Gebäude in Köln den deutschen Behörden. Ein Teil der israelischen Mitarbeiter der Einkaufsmission hatte sich mit Familie in der Bundesrepublik niedergelassen. Gemäß Weisung aus Jerusalem kapselten sie sich von ihrer deutschen Umgebung ab und lebten zurückgezogen. Die Kinder besuchten Schulen in Holland, eine

${ }^{8}$ Zum Aufbau der Israel-Mission: Shinnar an den Finanzminister, an Dr. G. Josephthal und an Hillel Dan betr. Bericht Nr. 3 "Organisation der Arbeit “ vom 15. 5. 1953, ISA, 2383/19.

${ }^{9}$ Interview mit A. Robert Sadove in Washington, D.C. im Frühjahr 1988.

10 Bericht zum Schilumimabkommen und dessen Umsetzung vom 30.6. 1962 [Original hebr.], durch Zyklostyl vervielfältigt, vom 10. 9. 1962, im Besitz des Autors.

11 Ebd. 
hohe emotionale und finanzielle Belastung für die Eltern. Die israelischen Diplomaten der Israel-Mission bekamen das feindselige Verhältnis der israelischen Regierung Deutschland gegenüber durch ihre Diskriminierung zu spüren. Sie erhielen niedrigere Gehälter als ihre Kollegen, die in einem anderen Land akkreditiert waren, ohne daß es dafür einen triftigen Grund gegeben hätte. Erst als die Leitung der Mission geschlossen zurücktrat, wurde ihr ein den örtlichen Verhältnissen angemessenes Entgelt versprochen. ${ }^{12}$ Die israelische Presse verfolgte die Tätigkeit der Einkaufsmission in Köln mit besonderer Aufmerksamkeit. Sie wurde regelmäßig kritisiert und gelegentlich verunglimpft. ${ }^{13}$ Die Kölner Mission und die Schilumimverwaltung in Israel waren zwar nicht frei von Betrugs- und Bestechungsfällen, hoben sich in dieser Hinsicht jedoch nicht von anderen öffentlichen Einrichtungen in Israel ab. ${ }^{14}$

Zum Chef der israelischen Einkaufsmission war kurz nach der Unterzeichnung des Luxemburger Abkommens Felix Eliezer Shinnar ernannt und mit deren Aufbau betraut worden. Seine Erfahrung machte Shinnar zum unumstrittenen Kanditaten für diesen Posten. Dem Urteil von Arbeitskollegen zufolge wies sein Charakter gewisse cholerische Züge auf. Bei fast jeder Meinungsverschiedenheit über wirtschaftliche oder politische Fragen soll er mit dem Rücktritt gedroht haben. Er wird als hart und ehrgeizig beschrieben, galt aber auch als hervorragender Fachmann auf seinem Gebiet. Meroz schilderte Shinnar als Chef, der von seinen Mitarbeitern gleichzeitig gefürchtet und verachtet wurde. Ein ehemaliger Sicherheitsoffizier der Mission bezeichnete ihn verächtlich als "Mann der Diaspora“ (im Gegensatz zum israelischen Ideal des "Sabre“, des in Israel geborenen Nachkommens jüdischer Einwanderer). ${ }^{15}$ Robert Sadove verwendete einen ähnlichen Ausdruck. ${ }^{16}$ Nahum Shamir, der dritte Direktor der Schilumimgesellschaft, stufte Shinnars Verhältnis zu den Deutschen als „unterwürfig“ ein. In fast abgöttischer Verehrung für Adenauer sei Shinnar den Worten des Kanzlers blindlings gefolgt und habe vor allem die Interessen des Auswärtigen Amts der Bundesrepublik berücksichtigt. ${ }^{17}$ In den Dokumenten des israelischen Außenministeriums wird Shinnar weder besonders gelobt noch kritisiert. Ben Gurion erwähnte ihn häufig in seinem Tagebuch. Politiker, Knessetabgeordnete und Journalisten fanden kaum gute Worte für ihn.

Ganz anders fiel die Bewertung mancher deutscher Beobachter aus. Eindeutig positiv war die Beurteilung durch Bundespräsident Theodor Heuss: „[Shinnar ist] ein Mann, der seiner schweren Aufgabe mit ruhigem Takt und viel Einsicht gerecht wird. " ${ }^{18}$ Nicht viel anders sind die Urteile über ihn in den Erinnerungen anderer deutscher Amtsträger ausgefallen. Felix von Eckardt schrieb über den Leiter der Israel-Mission: „Shinnar vertrat die Interessen seines Landes mit Klugheit und

12 Shinnar an den Finanzminister vom 27. 9. 1953, ISA, 572/2a.

13 MA'ARIv (Tel Aviv) vom 4./5. 6. 1956; KNESSET-PROTOKOLLE [Original hebr.], 3. Knesset, 166. Sitzung, S. 25, am 9. 10. 1956, 506. Sitzung, S. 2487.

14 Interview mit Nachum Shamir am 20. 5. und 17. 6. 1987.

15 Telephoninterview am 23.6.1993. Der Mann möchte nicht namentlich genannt werden.

16 Interview mit A. Robert Sadove in Washington, D.C. im Frühjahr 1988.

17 Interview mit Nahum Shamir am 20. 5. und 17.6. 1987.

18 PIKART, Theodor Heuss, S. 153. 
Umsicht, aber auch mit Verständnis für die deutsche Seite. "19 Hans von Herwarth bezeichnete Shinnar als "tüchtige und sympathische Persönlichkeit“. Shinnar sei frei von Ressentiments gegen Deutschland gewesen. ${ }^{20}$ Ähnlich äußerte sich im Rückblick auch der Israel nicht besonders freundlich gesinnte Alt-Bundespräsident Karl Carstens, der Shinnar als einen „geschickten, klugen Diplomaten“ bezeichnete. ${ }^{21}$ Ein nicht namentlich genannter Vertreter des Auswärtigen Amts schilderte Shinnar in einem zusammenfassenden Bericht anläßlich der Rückkehr des israelischen Diplomaten nach Israel im Jahre 1965 wie folgt: „SShinnar] hat sich die deutsch-israelische Aussöhnung zum Lebensziel gesetzt [...] [Er verbindet] die Zähigkeit des Schwaben mit der Subtilität und Intelligenz des jüdischen

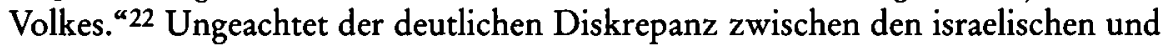
deutschen Beurteilungen gilt als unbestritten, daß Shinnar die israelische Einkaufsmission in Köln zwölf Jahre lang mit beachtlichem Erfolg - vor allem im wirtschaftlichen und finanziellen, weniger im politischen Bereich - geleitet hat. Im Juli 1953 verlieh ihm die israelische Regierung den persönlichen Rang des generalbevollmächtigten Gesandten und im Januar 1958 den Rang des Botschafters ad personam. Die westdeutschen Ämter respektierten Shinnars Titel in der Regel, gaben mitunter jedoch auch arabischem Druck gegen Shinnar nach.

Shinnar war ein einsamer Mann. Die Abende verbrachte er ganz allein in seiner Wohnung. Aus ethischen Gründen soll er auf geselligen Umgang mit Deutschen verzichtet haben, und aus demselben Grund hat sich seine Frau angeblich geweigert, nach Köln zu ziehen. Shinnar hatte freien Zugang zu führenden Politikern, einschließlich der Bundespräsidenten Heuss und Lübke und der Bundeskanzler Adenauer und Erhard. Mit dem Schwaben Heuss unterhielt er sich im gemeinsamen muttersprachlichen Dialekt. Shinnar neigte der CDU zu, ein Umstand, den die SPD und die Gewerkschaften mit einigem Mißfallen vermerkten. ${ }^{23}$

Die am 1. März 1953 gegründete Schilumimgesellschaft war für die praktische Durchführung des Abkommens zuständig. Ihr erster Direktor, Hillel Dan, amtierte gleichzeitig als Direktor des gewerkschaftlichen Baukonzerns Solel-Boneh, des Vorzeigeunternehmens der israelischen Einheitsgewerkschaft Histadrut. Die israelische Wirtschaft gliederte sich in einen öffentlichen Sektor, darunter staatseigene Betriebe, Gewerkschaftsfirmen und Kooperativen, und einen privaten Sektor mit Familienbetrieben und Aktiengesellschaften. Der Wettbewerb zwischen den beiden Sektoren, vor allem zwischen den Unternehmen der Histadrut und privaten Firmen in sämtlichen Wirtschaftsbereichen war nicht spannungsfrei. Während im öffentlichen Sektor die Mapai dominierte, galten die Parteien des Zentrums und der Rechten als Sprachrohr privater Interessen. Die Ernennung Dans zum Direktor der Schilumimgesellschaft verschaffte dem öffentlichen Sek-

19 ECKARDT, Lebenserinnerungen, S. 203.

20 Herwarth, Von Adenauer zu Brandt, S. 90.

21 CARSTENS, S. 307.

22 Bericht des Auswärtigen Amtes vom 1. 5. 1965, PA, IB4, B36, Nr. 192, 82.03-82.50, 92.12, Stichwort.

${ }^{23}$ Ludwig Rosenberg an Aharon Becker vom 9. 1. 1965, ILA, 219a/IV, No. 132; Heinz Putzrath an Erich Ollenhauer vom 3. 7. 1959, AdsD, SPD-Parteivorstand; Tavor an Herlitz vom 21. 3. 1960, ISA, 3309/10. 
tor einen Wettbewerbsvorteil, was zu ständigen Auseinandersetzungen über die Besetzung des Postens bis zum erzwungenen Rücktritt Dans im Jahre 1954 führte.

Der ehrgeizige und pragmatische Dan schien anfangs der geeignete Mann für den Aufbau und die Führung eines Unternehmens wie der Schilumimgesellschaft zu sein. Für die Unternehmensleitung rekrutierte er Führungskräfte von Histadrut-Firmen, für deren Gehälter weiterhin die bisherigen Arbeitgeber aufkamen. Dan formulierte Managementkonzepte und Leitlinien für die langfristige Wirtschafts- und Handelsstrategie im Geiste des Sozialismus, die er mit dem gesellschaftspolitischen Ethos der israelischen Arbeiterbewegung in Übereinstimmung $\mathrm{zu}$ bringen versuchte. Sie waren planwirtschaftlich orientiert, von oben verordnet und als Teil des öffentlichen Sektors konzipiert. Dans Auffassung zufolge, die auch vom dritten Direktor der Schilumimgesellschaft, Nahum Shamir, geteilt wurde, waren der Privatsektor bzw. private Unternehmen nicht in der Lage, größere Projekte auszuführen.

Die Debatte über die Verwendung der Schilumimgelder begann fast zeitgleich mit den Schilumimverhandlungen. In diesem Zusammenhang nahm die israelische Regierung die Dienste des amerikanischen Wirtschaftsplanungsexperten Samuel Trone in Anspruch, der am 30. Juni 1952 einen Bericht mit dem Titel „German Payments, Basic Development Program" vorlegte. ${ }^{24}$ Sein Plan wurde kurzerhand verworfen. Er offenbarte eine völlig falsche Einschätzung der Mentalität der Israelis, besonders der politischen, gesellschaftlichen und wirtschaftlichen Eliten. Trone betonte die private Initiative bzw. den Privatsektor. Seine Auftraggeber von der sozialistisch orientierten Mapai hatten offensichtlich einen ganz anderen Bericht erwartet. Andere Planer scheiterten auf ähnliche Weise. In Ermangelung einer langfristigen Planung griffen Dan und seine Nachfolger schließlich auf die bewährte israelische Methode der Improvisation zurück. Die einzelnen Ministerien notierten ihren Bedarf auf speziellen Fragebögen und bekamen eine bestimmte Summe zugeteilt. Private Industrielle reichten Bestellungen ein. Politiker und Beamte entschieden sich - nach sowjetischen Vorbild - für den Aufbau einer Schwerindustrie, einschließlich Eisen- und Stahlerzeugung, sowie Automobilund Dieselmotorenbau. Ein weiterer bevorzugter Verwendungsbereich für Schilumimgelder war der Ausbau von Verbindungs- und Kommunikationssystemen wie Eisenbahn, Straßennetz, Telekommunikation, Häfen und Rundfunk. Israel war zudem dringend auf den Ausbau der Stromerzeugung angewiesen. Von neuen Leichtindustrien erwartete man die Versorgung der Bevölkerung mit Konsumgütern sowie die Ankurbelung der Ausfuhr. Dieser Bereich war dem privaten Sektor zugedacht, der die in ihn gesteckten Erwartungen jedoch nicht erfüllen konnte. Statt mit Initiative und Kreativität tat sich dieser Sektor, wohl auch aus Mangel an Investitionskapital, vor allem mit Protest gegen sein angebliche Diskriminierung hervor. Die privaten Unternehmer, so Shamir, reichten nur ungenügend ausgearbeitete Geschäftspläne ein, da ihnen das nötige Fachwissen fehle. Die Verwendung der Schilumimgelder entwickelte sich zum Zankapfel zwischen

${ }^{24}$ Bericht von Samuel Trone „German Payments, Basic Development Program“ vom 30.6. 1952, BGA, General File. 
den linken Parteien und dem restlichen Parteienspektrum, das vom Privatsektor unterstützt wurde. ${ }^{25}$

Der Nettoerlös aus dem Verkauf von Schilumimgütern wurde auf die verschiedenen Wirtschaftszweige verteilt. Größere Summen gingen in die Forschung und Entwicklung, ein anderer Teil der Gelder floß in die Verteidigung, in die Entwicklung von Waffen und in die Rüstungsindustrie. ${ }^{26}$ Wirtschaftsfachleute forderten, das angehäufte Kapital zur Festigung der Landeswährung zu verwenden. Die israelische Wirtschaft befand sich in einer Inflationsspirale, das israelische Pfund verlor ständig an Wert, und die weltwirtschaftlichen Einflüße waren, bedingt durch den Kalten Krieg, negativ. Der Wechselkurs der Schilumimmark war zudem umstritten und häufiger Neuanpassung unterworfen. Die Finanzexperten traten deshalb für die Schaffung eines Fremdwährungsfonds zur Stabilisierung des israelischen Pfunds ein. Doch die Versuche, die deutsche Seite davon zu überzeugen, einen Teil der Schilumim in bar und nicht in Form von Gütern zu leisten, schlugen fehl, und damit scheiterte auch der Stabilisierungsplan. Die Schilumimgesellschaft verfolgte die Warenmärkte aufmerksam, um Preisschwankungen auszunützen. Rohstoffe wurden zu tiefen Preisen gekauft und später mit Profit verkauft. Die Einfuhr von Konsumgütern für den täglichen Bedarf aus Schilumimgeldern war strikt untersagt, davon ausgenommen nur der Import von Hering, Zucker, Kaffee, Fleisch und Kakaobohnen. Trotz Wirtschaftsplanung und detaillierten Empfehlungen für die Verwendung der Gelder, setzten die Beamten weitgehend auf Improvisation.

Ein Hauptgrundsatz des Schilumimabkommens war zum Schutz der lokalen Industrie vor ausländischer Konkurrenz das Einfuhrverbot von Gütern, die auch in Israel hergestellt wurden. Dies führte in einigen Fällen zu Versorgungsengpässen bzw. zu einem qualitativ minderwertigen Angebot zu überhöhten Preisen, ein Mißstand, dem mehrere Knessetsitzungen gewidmet waren. ${ }^{27}$ Ein weiteres Problem war die Bevorzugung deutscher Erzeugnisse - gegen Schilumimgelder - auf Kosten traditioneller Lieferanten aus Großbritannien, Frankreich und anderen Ländern. Die traditionellen Lieferanten protestierten gegen diese neue Handelspolitik über diplomatische Kanäle oder mittels Druck via Firmen in jüdischem Besitz sowie über jüdische und zionistische Gruppierungen und Organisationen. In den meisten Fällen entschied sich der Staat Israel jedoch für den wirtschaft-

25 Interview mit Nachum Shamir am 20. 5. und 17. 6. 1987; KNESSET-PROTOKOLLE [Original hebr.], 2. Knesset, 242. Sitzung, S. XIV, Dr. Yeshayahu Foerder, die Fortschrittspartei, zum Haushaltsplan für das Jahr 1954/55 am 8. 6. 1953; der Berater an den Außenminister vom 26. 3. 1954, USNA, National Record Center Suitland, RG 466, HICOG, Bonn, Box 169.

26 Tagebucheinträge Ben Gurions vom 14. 2. und 1. 10. 1952, BGA, BGD.

27 KNESSET-PROTOKOLLE [Original hebr.], 2. Knesset, 356. Sitzung am 18. 1. 1954, 447. Sitzung am 28.6. 1954, 451. Sitzung am 30. 6. 1954, 467. Sitzung am 9. 8. 1954, 508. Sitzung am 7. 9. 1954 und 588. Sitzung am 1. 12. 1954; E.J. Barnes, Bonn, an F.A. Warner, London, vom 26. 7. 1959, PRO, FO 371/118407, WG 1571; Evant, Tel Aviv, an Eden, London, vom 22. 9. 1952, PRO, FO 371/98802; Ausarbeitung „Deutsche Wiedergutmachungsleistungen an Israel und deren Auswirkung auf Großbritannien vom 30. 4. 1953, ISA, 45/9; Shinnar an den Finanzminister vom 4. 10. 1953, ISA, 588/4; DÜSSELDORFER NACHRICHTEN vom 3. 8. 1953. 
lichen Vorteil, d.h. für die kurzfristige Einsparung von Devisen, wenn auch im Hinblick auf die langfristigen Risiken versucht wurde, den Schaden zu begrenzen.

Verschiedene Staaten versuchten von den Schilumim zu profitieren; sei es, um mittels Ausfuhr nach Israel Schulden gegenüber der Bundesrepublik abzutragen oder schlicht um den Handel mit Israel - gegen Schilumimgelder - zu fördern. Hierbei handelte es sich im wesentlichen um die in Israel nicht unumstrittenen Dreiecksgeschäfte. Der jüdische Staat importierte auf diesem Weg Waren, die in der Bundesrepublik nicht erhältlich waren, darunter Rohgummi, Häute, Kakao, Kaffeebohnen, Bauholz, Zucker und Weizen. Solche Geschäfte, in manchen Fällen unumgänglich und oft auf Drängen der israelischen Regierung zustande gekommen, erwiesen sich nicht selten als Fehlschlag. So zwang die Bundesrepublik Israel zum Kauf von türkischem Weizen, der sich als qualitativ minderwertig und zu teuer herausstellte. Die Türkei hatte Schulden bei der Bundesrepublik, und für Bonn bot sich die Möglichkeit, zwei Fliegen mit einer Klappe zu schlagen. ${ }^{28}$ Bei einem ähnlichen Geschäft belieferte Österreich Israel im Auftrag der Bundesrepublik mit schlechtem Holz.29 Nur widerstrebend wurde die Einfuhr von westdeutschen Fischprodukten akzeptiert, nachdem sich der israelische Konsument an den qualitativ besseren skandinavischen Fisch gewöhnt hatte. Israel weigerte sich sodann, Schilumimgelder für die Einfuhr von isländischem Fisch zu verwenden, wie von der Bundesrepublik verlangt, denn den konnte es im Tausch gegen Zitrusfrüchte erhalten. Kurz, Israel verfolgte die Warenmärkte mit größter Aufmerksamkeit und war nicht bereit, die eigenen Handelsinteressen auf dem Altar der Schilumim zu opfern. Aber auch die Bundesregierung bewilligte Dreiecksgeschäfte nur in Ausnahmefällen und untersagte sie nach 1956 ganz. Die Förderung der eigenen Industrie hatte auch für die Bundesrepublik oberste Priorität. ${ }^{30}$ Größte Bedeutung wurde in diesem Zusammenhang dem Wiederausfuhrverbot beigemessen ${ }^{31}$, dessen Einhaltung von deutschen Diplomaten streng überwacht wurde. Arabische Diplomaten werteten Dreiecksgeschäfte als Übertretung des Wiederausfuhrverbots. 32

Das Schilumimabkommen unterteilte die zu liefernden Güter in fünf Kategorien mit festem Anteil an der Jahresrate nach folgendem Schlüssel: Eisen und farbige Metalle: 43 Millionen Mark, Leichtindustrieprodukte: 47 Millionen Mark,

${ }^{28}$ Shinnar an den Finanzminister vom 6. 8. 1953; MA'ARIV (Tel Aviv) vom 7. und 11. 8. 1953.

29 MA'ARIV (Tel Aviv) vom 19. 5. 1954; Memo vom 7.7. 1954, PRO, FO 371/104799, ER11318/3.

30 Bartur, Wirtschaftsabteilung, an Shinnar vom 30. 8. 1953, ISA, 2417/8; Levavi an Yoran, Belgrad, vom 25. 5. 1953, ISA, 2418/11a; Dr. Brückner an alle Außenposten der BRD vom 17. 8. 1954, PA, 210-01/E, Bd. 8; Bundesfinanzminister Schäffer an Staatssekretär Wolff vom 6. 8. 1953, BArch, B 125/51545; Notiz vom Juli/August 1957, PRO, FO 371/128172.

31 Documents Relating, Par. 5, Punkt 4, S. 127.

32 Von Grundless an das AA vom 25. 2. 1952, PA, 210-01/25, Bd. 1; Vermerk über den Besuch des irakischen Geschäftsträgers Mumayin im AA vom 21. 2. 1956, PA, 708, 82.2082.70,92.10; Bundeswirtschaftsministerium, Beelitz, an Abs vom 22.3. 1952, BArch, B 702/7019; Bundesfinanzministerium, Schäffer, an den Staatssekretär vom 18. 11. 1953, BArch, B 126/51545; Yachil an den israelischen Außenminister vom 8. 8. 1953, ISA, 617/ 16; Ilsar an Tavor vom 24. 6. 1957, ISA, 3100/11b. 
Maschinen: 40 Millionen Mark, landwirtschaftliche Güter: 30 Millionen Mark, Dienstleistungen: 15 Millionen Mark und Mineralöl: 75 Millionen Mark. Zusammen ergab das einen Betrag von jährlich 250 Millionen Mark. ${ }^{33}$ Die Israelis beklagten die "Sturheit" der deutschen Seite, die sich weigerte, die festgelegten Gütergruppen, das Verhältnis zwischen ihnen oder den Zahlungsmodus zu ändern. ${ }^{34}$ Diese Haltung stieß bei den unternehmerisch-dynamischen, improvisierenden Israelis auf wenig Verständnis und wurde manchmal als Böswilligkeit interpretiert. ${ }^{35}$ Am Anfang konzentrierten die Israelis ihre Bestellungen jedes Jahr auf eine bestimmte Kategorie. Sie bestellten ganze Fabriken, sehr zum Mißfallen der deutschen Seite, da solche Anlagen, sogenannte harte Güter, auf dem Weltmarkt hohe Preise erzielten. Die Bundesrepublik zog es vor, Israel „weiche Güter" zu liefern, die auf dem normalen Markt schwieriger bzw. nur mit geringem Profit verkäuflich waren. Bei den "harten Gütern" handelte es sich in der Regel um hochprofitable Produkte der Schwerindustrie mit ausgezeichneten Märkten. ${ }^{36}$ Zur dritten Produktgruppe, die in Israel großes Interesse weckte, gehörten dringend benötigte Dünger und Rohstoffe für die Chemie sowie halbfertige Produkte für die Gummi- und Textilindustrie. Die vierte Produktkategorie war umstritten, denn die darin enthaltenen Konsumgüter widersprachen den allgemeinen Richtlinien für die Verwendung von Schilumimgeldern. Sie veranschaulicht freilich, wie die westdeutsche Wirtschaft von den Schilumim profitierte. Deutsche Winzer übten Druck auf die deutsche Schilumimverwaltung aus, um die Einbeziehung von Weinen in das Lieferprogramm zu erwirken, was von der israelischen Seite jedoch abgelehnt wurde. Wein galt in Israel als Luxusartikel, der im Land selbst hergestellt wurde. Der deutsche Wein war zudem nicht koscher und somit für gläubige Juden tabu. Nach heftiger Diskussion ließt die deutsche Seite diesen Posten fallen. Auch die fünfte Güterkategorie war mit Meinungsverschiedenheiten über die Aufteilung des Transports zwischen israelischen und deutschen Reedereien und des Versicherungsgeschäfts verbunden. Die Zusammenstellung der Güterlisten war generell vom Gegensatz zwischen der israelischen Bevorzugung „harter Güter" und dem deutschen Interesse an der Lieferung "weicher Güter“ geprägt. Ein auf arabischen Druck zurückzuführender angedrohter Lieferstopp an Israel in den frühen sechziger Jahren konnte nur durch eine Intervention von hoher Stelle verhindert werden. ${ }^{37}$

Die Bevorzugung der sogenannten Notstandsgebiete bei der Güterbestellung leistete einen bedeutenden Beitrag zur Entwicklung der westdeutschen Wirtschaft. Davon profitierte - gemäß Abkommen - vor allem West-Berlin bzw. Berliner Firmen im Telekommunikationsbereich. Weitere Schwerpunkte der israelischen Bestellungen lagen in Niedersachsen, Schleswig-Holstein, Hamburg und Bremen. Die Bundesländer Niedersachsen und Schleswig-Holstein wurden als

33 LAZARSOHN, Umsetzung, S. 78.

34 Interview mit Nahum Shamir am 20. 5. und 17.6. 1987; Vermerk der Abteilung 206 vom 29. 11. 1955, PA, 210-01/E.

35 Interview mit Nahum Shamir am 20. 5. und 17.6. 1987.

36 ChEVRATChEVRAT HA-SHILUMIM.

37 Aufzeichnung vom 8. 8. 1960, PA, Nr. 1032, 308, 82.50, 92.19, Ref. 416. 
Grenzgebiete zur DDR bevorzugt behandelt, und in den Hansestädten Hamburg und Bremen gab es Werften, die seit dem Krieg brachlagen. Israelische Bestellungen von großen Frachtern hauchte ihnen und den Werften in Lübeck neues Leben ein. Der Ausbau der israelischen Handelsflotte war ein Kapitel für sich. Trones Pläne maßen der Flotte niedrige Priorität bei. Sowohl Dan als auch Shamir beanspruchen den Ausbau dieses Wirtschaftszweigs in ihren Erinnerungen für sich. ${ }^{38}$ Übereinstimmend stellen sie indes fest, daß die israelischen Reedereien die sich ihnen bietende Gelegenheit nicht wahrgenommen hätten und Finanzminister Eschkol für den Ausbau der Handelsschiffahrt, Handelsminister Saphir aber dagegen gewesen sei. Eschkol soll argumentiert haben, überzählige Schiffe seien leicht zu verkaufen. Insgesamt ließ Israel 59 Schiffe unterschiedlicher Art und Größe bauen, die den bundesdeutschen Werften zu einem Neuanfang verhalfen. ${ }^{39}$ Shamir weist auf den Beitrag der israelischen Bestellungen zur technologischen Entwicklung der westdeutschen Industrie hin und führt als Beispiel die Turbinenbestellung für das Haifaer Elektrizitätswerk an, die die Ingenieure veranlaßt haben soll, sich in die Neuerungen des Turbinenbaus zu vertiefen. Obwohl die Schilumim selbst in den Spitzenjahren weniger als ein Prozent des Bruttosozialprodukts der Bundesrepublik ausmachten, hat die westdeutsche Wirtschaft zweifellos davon profitiert, wovon das anfängliche rege Interesse der Industrie an Schilumimaufträgen zeugt. ${ }^{40} \mathrm{Die}$ Schilumim trugen zudem nicht unwesentlich zur Lösung sozioökonomischer Probleme in der Bundesrepublik bei. Doch mit der Ausdehnung der wirtschaftlichen und industriellen Tätigkeit und dem Aufkommen der arabischen Erpressungsversuche verflog die Begeisterung bald.

Israel bot den westdeutschen Exporteuren einen neuen und rasch wachsenden Markt, zu dem sich manche Hersteller durch die Schilumim langfristig Zugang verschafften. Am Anfang konzentrierte sich der Handel auf Ersatzteile für deutsche Maschinen, später erweiterte sich das Sortiment. Als die Bundesrepublik und der Staat Israel im Jahre 1965 nach Abschluß der Schilumimlieferungen diplomatische Beziehungen aufnahmen, führte Israel bereits westdeutsche Güter im Gesamtwert von 276 Millionen Mark ein. Die israelischen Ausfuhren in die Bundesrepublik betrugen zum selben Zeitpunkt 206 Millionen Mark. Die Bundesrepublik Deutschland war zum drittwichtigsten Handelspartner Israels aufgestiegen.

Der Schilumimimport war nicht nur eine Erfolgsgeschichte. Gelegentlich kam es zu Fehleinschätzungen des eigenen Bedarfs oder zu anderen groben Entscheidungsfehlern. Ein solcher Fall betraf die riesigen Investitionen in Kupferminen bei Elat am Roten Meer. Erst nach Beendigung der Bauarbeiten stellten die Unternehmer fest, daß der Preis des Endprodukts weit über dem Weltmarktpreis lag.

38 DAN, Ungebahnte Wege, S. 333-335; Interview mit Nahum Shamir am 20. 5. und 17. 6. 1987.

39 DeutschlandS Weg Nach IsRael, S. 101.

40 BUNDESANZEIGER vom 12. 6. 1952; Shinnar an Eytan vom 6. 6. 1953, ISA, 568/3; Ausarbeitung „Das Interesse der westdeutschen Industrie und Berlins an den Lieferungen aus dem Wiedergutmachungsabkommen mit dem Staat Israel" vom 12. 2. 1953; Auszug aus der Liste Industrie-Angebote an die Israel-Mission betr. Lieferungen unter dem Wiedergutmachungsabkommen mit Israel vom 10. September 1952, BArch, N 1351, Bd. 16. 
Schlicht größenwahnsinnig waren sodann Bestellungen für Eisenbahneinrichtungen und Rollmaterial. Die verantwortlichen israelischen Beamten hatten die Warnungen des deutschen Herstellers, wonach sich das bestellte Material für die kurzen Distanzen in Israel nicht eigne, kurzerhand in den Wind geschlagen und scheiterten kläglich. ${ }^{41}$ Als das Schilumimprogramm 1965 formell zu Ende ging, war der Etat längst erschöpft. Aus Mangel an Vertrauen zur deutschen Seite hatten sich die Israelis bemüht, den Etat so schnell wie möglich aufzubrauchen. Sie tätigten Großaufträge mit langfristigen Lieferterminen, finanziert durch langfristige Kredite der beauftragten Firmen selbst oder durch Israel freundlich gesinnte Banken. Holländische und später auch deutsche und israelische Banken gewährten Kredite zu niedrigen Zinsen, die später von der Bank deutscher Länder bzw. der Deutschen Bundesbank zurückgezahlt wurden. Das sogenannte Vorgriffsystem machte es möglich, den Schilumimetat viel schneller als vorgesehen zu nutzen. Dadurch waren am 31. März 1956 bereits Bestellungen für 45,5\% der gesamten Schilumimsumme vergeben. 42

Die Schilumimgesellschaft in Israel setzte sich aus drei Organen zusammen: aus der Exekutive einschließlich des für die Übermittlung von Anweisungen an die Kölner Mission und die Handelsabteilung verantwortlichen technischen Ausschusses, einem öffentlichen Aufsichtsrat bestehend aus 34 Mitgliedern sowie aus einem ministeriellen Aufsichtskomitee.

Die Aufgabe der Schilumimgesellschaft bestand darin, für den Transport der bestellten Güter nach Israel zu sorgen. Sie war verantwortlich für die Ausführung der eigenen und fremden Bestellungen sowie für die Auslieferung der bestellten Güter in Israel. Die Exekutive war für die Verwaltung zuständig. Die Bestellungen wurden dem technischen Ausschuß zur Prüfung vorgelegt und bei einer positiven Entscheidung an die Mission weitergeleitet, der es oblag, einen geeigneten Hersteller zu ermitteln. War der Hersteller gefunden, mußte sich der Käufer um die, Einfuhrbewilligung des zuständigen Ministeriums bemühen. Gleichzeitig waren entsprechende Vorkehrungen für die Finanzierung des Auftrags zu treffen. Der Käufer hatte 12,5\% des Auftragswerts im voraus zu bezahlen. Weitere $25 \%$ waren bei der Freigabe der Ware im Hafen fällig. Für den Rest wurde ein sechsmonatiger Kredit gewährt. Nach gesicherter Finanzierung wurde der Auftrag von der entsprechenden Abteilung der Kölner Mission zur Ausführung freigegeben. Von dort gelangte er direkt an den Hersteller. Vermittler oder Handelsagenten waren nicht zugelassen. ${ }^{43}$ Ben Gurion und andere israelische Stellen hatten sich ausdrücklich für den Ausschluß von Handelsagenten entschieden - eine Haltung, die von deutscher Seite mitgetragen wurde. Eine enstprechende Bestimmung ist in Absatz 7 des Schilumimabkommens enthalten. Ben Gurion hatte seine Auffassung damit begründet, daß bei einer Zulassung von Handelsagenten eine Überschwemmung der Märkte durch israelische Geschäftemacher zu befürchten

41 Interview mit Nachum Shamir am 20.5. und 17.6.1987.

42 ChevratChevrat ha-SHILumiM, S. 7; Interview mit Nahum Shamir am 20. 5. und 17. 6. 1987; LAZARSOHN, Umsetzung, S. 77.

43 LAZARSOHN, Umsetzung, S. 76-77; Aufzeichnung vom 17. 11. 1952; Pressekommuniqué Shinnars vom 22. 3. 1953, PA, 244-13E II, 15315/52-1748/52. 
sei. ${ }^{44}$ Interessierte Kreise hatten alle Hebel in Politik, Presse und Wirtschaft in Bewegung gesetzt, um eine Änderung dieser Bestimmung zu erzwingen. ${ }^{45}$ Der öffentliche Aufsichsrat, bestehend aus Vertretern der Regierung, des öffentlichen Lebens und verschiedener Wirtschaftszweige war für Grundsatzfragen zuständig. Häufige Tagesordnungspunkte waren der Wettbewerb zwischen dem öffentlichen und dem privaten Sektor sowie Klagen über Diskriminierungen. Die höchste Entscheidungs- und Aufsichtsgewalt lag bei dem vom Finanzminister geleiteten ministeriellen Aufsichtskomitee, dem auch der Landwirtschaftsminister, der Minister für Industrie und Handel, der Minister für Arbeit und der Innenminister angehörten. Ein Planungsausschuß und die Leitung der Schilumimgesellschaft standen ihm zur Seite. ${ }^{46}$

Die israelischen Stellen arbeiteten eng mit den deutschen Institutionen zusammen, vor allem mit dem Auswärtigen Amt, dem Bundesministerium der Finanzen und dem Bundeswirtschaftsministerium.

\section{Die ausführenden Organe der Bundesrepublik}

Die genannten Bundesministerien waren für Grundsatzfragen zuständig, wurden aber auch bei kleineren Problemen konsultiert. Daneben beteiligten sich weitere Stellen und Persönlichkeiten des öffentlichen Lebens an der Umsetzung des Schilumimabkommens. Der Aufbau der deutschen ausführenden Organe ist in den Absätzen 5, 6 und 7 des Schilumimabkommens definiert. ${ }^{47}$ Hier ist zunächst der gemischte Ausschuß zu erwähnen, der sich aus Delegierten beider Seiten zusammensetzte. Den Vorsitz nahm der erste deutsche Chefdelegierte, Carl Hermann Müller-Graff, ein hochrangiger und einflußreicher Vertreter des Bundeswirtschaftsministeriums, ein. Die Israelis waren mit dieser Besetzung sehr zufrieden und bedauerten Müller-Graffs Abberufung im November 1953. Er galt als Fachmann, stand dem Schilumimabkommen und dem Geist, auf dem es beruhte, positiv gegenüber und war als ehemaliger Gegner der Nationalsozialisten bekannt. ${ }^{48}$ Der gemischte Ausschuß trat am 18. Mai 1953 zu seiner konstituierenden Sitzung zusammen. Es wurden vier Unterausschüsse für folgende Bereiche gebildet: Berlin und die Notstandsgebiete, Transportfragen, Unkosten (Kommissionsgebühren, Verwaltungskosten, Versicherung usw.) und Finanzierung. Der gemischte Ausschuß hatte den Auftrag, die Warenliste jeweils bis spätestens zum 30. Sep-

44 Ben Gurion an Goldmann vom 2. 8. 1952, CZA, Z 6/1022.

45 Von Carnap, Außenhandelsabteilung des Bundesverbands der Deutschen Industrie, an Dr. A. Bergmann, Israel-Mission, vom 3. 12. 1954, ISA, 613/12; KNESSET-ProTOKOLlE [Original hebr.], 3. Knesset, 506. Sitzung am 4. 8. 1958, S. 2615-2626 (Resümee des Finanzausschusses der Knesset zur Frage von Handelsagenten und zum Kauf von SchilumimWaren).

46 Protokoll Nr. 313/3 über die Regierungssitzung vom 12.10. 1952, ISA, 7264/7; LAZARSOHN, Umsetzung, S. 76; der britische Botschafter in Tel Aviv an Anthony Eden vom 8. 11. 1952, PRO, FO 371/98802, ER 11318/5.

47 Documents Relating, Par. 5,6 und 7, S. 127-129.

48 Shinnar an den Wirtschaftsminister, an Dr. G. Josephthal und an Hillel Dan betr. Bericht Nr. 18 vom 4. 10. 1953, ISA, 2383/19. 
tember zu empfangen und sie binnen dreier Monate zu genehmigen. Der Vorschlag für das folgende Jahr hatte auf der Liste des Vorjahres zu beruhen, gewisse Änderungen waren jedoch möglich. Genau diese Änderungen verursachten die meisten Diskussionen und Meinungsverschiedenheiten und bildeten die Grundlage für die israelischen Klagen über angebliche Sturheit und Böswilligkeit der anderen Seite. Die israelischen Vertreter stellten sich auf den Standpunkt, daß sobald ein Posten auf der Liste der gestatteten Güter erschöpft sei oder Israel seine Prioritäten ändere, dies von der deutschen Seite nicht angefochten werden könne, lege doch das Abkommen ohnehin die genauen Lieferbedingungen, den gesetzlichen Status der Güter (d.h. Besteuerung, Zölle, Ausfuhrpriorität und Kaufbedingungen) sowie die Preise unter Berücksichtigung der lokalen und internationalen Märkte fest. Es sei demnach nicht Sache des Ausschusses, so die Israelis, neue Hürden aufzubauen. Doch die deutschen Vertreter waren anderer Meinung. Sie konsultierten verschiedene Stellen, bevor sie die vorgelegte Liste genehmigten. $\mathrm{Da}$ es die Hauptaufgabe des gemischten Ausschusses war, über die Zulässigkeit der einzelnen Bestellungen zu entscheiden, war die Genehmigung der Güterliste mit ausgedehnten Verhandlungen verbunden. Die genehmigte Liste wurde jeweils dem Bundesfinanzministerium zur Budgetierung vorgelegt.

Als nächtes legte die israelische Einkaufsmission die Kaufaufträge der Auftragsvergabestelle, einem speziellen Amt für die Verrechnung und Vergabe von Schilumimaufträgen, vor, nachdem die Handelsabteilung der Mission die nötigen Abklärungen getroffen hatte. Der Einkauf fand in enger Zusammenarbeit mit der Bundesstelle für den Warenverkehr der gewerblichen Wirtschaft in Frankfurt am Main statt, die mit der westdeutschen Industrie bzw. mit der Erhältlichkeit bestellter bzw. gewünschter Güter vertraut war. Diese Bundesstelle war außerdem verantwortlich für die fachgerechte Ausführung der Aufträge und die Gewährung der den israelischen Käufern zustehenden Privilegien. Zur Verhinderung von Preismanipulationen und zur Sicherstellung fairen Wettbewerbs holte die Stelle bei mehreren Herstellern Angebote ein. Generell wachte die Bundesstelle für den Warenverkehr über die Wahrung der Interessen sowohl der Käufer als auch der Anbieter. ${ }^{49}$

Dank ihrer Erfahrung und den engen Verbindungen zu verschiedenen Wirtschafts- und Industriekreisen war die Bundesstelle für den Warenverkehr in der Lage, auf eine reibungslose Zusammenarbeit und Koordination zwischen der Israel-Mission, den Herstellern und den Exporteuren hinzuwirken. Bereits während der Verhandlungen in Wassenaar hatte das Bundeswirtschaftsministerium den sogenannten Israel-Ausschuß unter Beteiligung des Industrie- und Handelstages in Bonn, des Bundesverbandes der Deutschen Industrie und eigener Vertreter ins Leben gerufen. Auf einer Sitzung vom 16. Juli 1952 empfahlen die sechs anwesenden Vertreter die Gründung eines speziellen aus Vertretern sämtlicher wichtiger Wirtschaftsverbände zusammengesetzten Gremiums, dessen Aufgaben wie folgt festgelegt wurden: Herstellung von Verbindungen zwischen israelischen

49 Bericht über die Beratungen im Bundeswirtschaftsministerium in Bonn am 14. 11. 1952, CZA, S 35/234; Memorandum vom 25. 11. 1952, CZA, S 35/252; Shinnar an den Finanzminister, an Dr. G. Josephthal und an Hillel Dan betr. Bericht Nr. 3 vom 15. 5. 1952, ISA, $2383 / 19$. 
Käufern und deutschen Anbietern, Beratung interessierter Firmen und Preisüberwachung - zwecks Verhinderung von Dumping, Garantierung des Sonderstatus von Berlin, Einhaltung der Verkaufsgrenzen und Kontrolle der Tätigkeit der Bundesstelle für Warenverkehr. ${ }^{50} \mathrm{Da}$ sich die wirtschaftliche Situation der Bundesrepublik nach der Abfassung dieses Dokuments nachhaltig verbesserte, erübrigte sich ein Teil der beschriebenen Aufgaben, andere wurden den neuen Verhältnissen angepaßt. Jedenfalls veranschaulicht das Dokument das rege Interesse der Industrie und der deutschen Wirtschaft generell an den Schilumim und die gründlichen Vorbereitungen des Projekts.

Die Bundesstelle für den Warenverkehr wurde auch beauftragt, die Preise zu überwachen und unfairen Wettbewerb zu verhindern sowie die Interessen der einzelnen Bereiche der westdeutschen Wirtschaft und der Einzelfirmen zu wahren. Den Berichten der Kölner Mission an das Finanzministerium in Jerusalem zufolge nahm die Bundesstelle ihren Auftrag bis zum Ende der Schilumim wahr. Ihre Hauptaufgabe war die Prüfung und Bewilligung der eingereichten Bestellungen. Insbesondere sollte geprüft werden, ob die bestellten Güter in der Warenliste enthalten waren, ob sie den vereinbarten Bedingungen entsprachen und ob es sich nicht um verbotenes Kriegsmaterial handelte. Bewilligte Bestellungen wurden den Anbietern zur Ausführung übergeben. Nach der Lieferung der Güter wurde die Bestellung, falls nicht anders vereinbart, der Bank zur Verrechnung überstellt.

Gemäß Absatz 9 des Schilumimabkommens, war die Israel-Mission dazu verpflichtet, bei der Bank deutscher Länder ein Konto zu eröffnen, auf welches die Bundesregierung $z$ weimal jährlich die vereinbarte Rate einzuzahlen hatte. ${ }^{51} \mathrm{Zu}$ Beginn ihrer Tätigkeit eröffnete die Mission fünf Konten bei westdeutschen Banken und später weitere Konten zur Bezahlung der ausgeführten Bestellungen.

Zur Verschiffung bereite Güter wurden zum Ausgangshafen transportiert. Befand sich dieser in der Bundesrepublik, ging der Landtransport vollumfänglich auf Kosten der Schilumim, der Landtransport zu ausländischen Häfen dagegen nur bis zur deutschen Grenze. Rund 52\% der Schilumimfracht fuhr unter israelischer Flagge, der Rest auf deutschen Schiffen oder auf von deutschen Reedereien gecharterten ausländischen Frachtern. ${ }^{52}$ Seetransport und Versicherung (cif) gingen ebenfalls auf Rechnung der Schilumim. 70\% des Risikos bzw. der Prämie entfielen auf ein Konsortium deutscher Versicherungen. Den Rest finanzierte ein israelisches Versicherungskonsortium, das wiederum bei einer englischen Gesellschaft rückversichert war und somit nur einen Bruchteil der restlichen Prämie zu zahlen hatte. Die Transportkosten gingen auf das Konto der fünften Schilumimgüterkategorie. ${ }^{53}$

Die Schilumim bildeten den Anknüpfungspunkt für die Beziehungen (oder besser gesagt der „Nicht-Beziehungen“") zwischen der Bundesrepublik Deutschland und dem Staat Israel. Die beiden Staaten formalisierten ihre bilateralen Beziehungen durch die Aufnahme diplomatischer Beziehungen genau zu dem Zeit-

\footnotetext{
50 Vermerk vom 17.7. 1952, BArch, B 102/7019.

51 Documents Relating, S. 126, 130-131.

52 ChevratChevrat ha-Shilumim, S. 7; Der deutsch-israelische Dialog, S. 126.

53 Documents Relating, Par. 6, Punkt d, S. 128.
} 
punkt, als die Schilumim zu Ende gingen, d.h. nach Ablauf der zwölfjährigen Zahlungsphase (1953-1965). Ohne diese Brücke wäre die Aufnahme diplomatischer Beziehungen zwischen der Bundesrepublik und Israel wohl kaum zustandegekommen. Eine These, die nur von wenigen Beobachtern offen unterstützt wird, besagt, daß der Abschluß der Schilumim die Hauptursache für die Formalisierung der gegenseitigen Beziehungen gewesen sei. Gegner der Normalisierung meinten, die Schilumim seien der beste Beweis dafür, daß Geld den Weg zu gegenseitigen Beziehungen geebnet habe. Sie betrachteten die Schilumim als Zeichen des moralischen Bankrotts: Das Andenken an die Ermordeten sei für Mammon veräußert worden. Andere werteten die strikte Einhaltung des Schilumimabkommens als Beweis dafür, daß sich die deutsche Bevölkerung seit dem Kriegsende nachhaltig geändert habe. Zahlreiche Stimmen in der Bundesrepublik, darunter auch von Spitzenpolitikern und hochrangigen Diplomaten, gaben ihrer Verwunderung darüber Ausdruck, daß von jüdischer Seite weiter moralische Ansprüche erhoben würden, trotz der Milliarden, die ihr zugeflossen seien. Sehr verbreitet war sodann in Deutschland die Meinung, daß der Holocaust mindestens zum Teil durch Schilumimgüter wieder-gut-gemacht werden konnte. Auch in Israel gab es Stimmen, die den Beitrag der Schilumim zur wirtschaftlichen Entwicklung, zur Verteidigung und zur Eingliederung von Neueinwandern in Israel würdigten und für die Aufnahme diplomatischer Beziehungen eintraten. Kein Zweifel, die Schilumim waren zu keiner Zeit bloß Wirtschaftsfaktor, sondern stets auch von philosophisch-ethischen Aspekten begleitet und insofern unzertrennlich mit der Frage der diplomatischen Beziehungen verbunden. Die gegenseitige Anerkennung und der Austauschs von Diplomaten war weit mehr als ein formaler Akt. Für manche Israelis war das neue Verhältnis zur Bundesrepublik Deutschland ein dramatisches Ereignis von immenser emotionaler Tragweite, während in der Bundesrepublik diplomatische Beziehungen zum jüdischen Staat oft lediglich als Frage der Staatsräson eingestuft wurden. Zwölf Jahre lang flossen Schilumimgüter nach Israel und dienten dabei als Vorwand, Rechtfertigung oder Deckmantel für die (Nicht-)Beziehungen zwischen beiden Staaten.

\section{Auf verschlungenem Weg zu diplomatischen Beziehungen}

Das Luxemburger Abkommen erfüllte die unmittelbaren wirtschaftlichen Erwartungen sowohl hinsichtlich der Schilumim als auch der individuellen Entschädigung, doch es konnte das israelische Mißtrauen gegen die deutschen Vertragspartner nicht ganz zerstreuen. „Sie werden nicht zahlen“, war eine verbreitete Meinung in Israel, nicht nur bei der rechten und linken Opposition, sondern auch in der Koalition und selbst in der Führung der regierenden Mapai. ${ }^{54}$ Sie verstummte erst, als der Güterstrom zu fließen begann.

${ }^{54}$ So z.B. Eliezer Livneh (Mapai) am 16.6. 1953 vor der Knesset: KNESSET-ProTOKOLLE [Original hebr.], 2. Knesset, 249. Sitzung, S. 1612; DAN, Ungebahnte Wege, S. 333-335; Presse- und Informationsdienst der Bundesregierung, dpa-Information vom 9. 4. 1953, PA, 210-01/E, Bd. 8. 
Allmählich wurden neue Kontakte geknüpft, die mit dem deutsch-israelischen Abkommen und den Schilumim nur noch am Rande zusammenhingen. In Wassenaar war zwar nicht über diplomatische Beziehungen verhandelt worden, doch auf israelischer Seite erkannte man bald, daß das Thema Anerkennung nicht mehr aus der Diskussion zu bringen war. Auf Anregung von Sharett vertrat Shinnar vor dem Knessetausschuß für Sicherheit und auswärtige Angelegenheiten den Standpunkt, daß das Abkommen faktisch eine gegenseitige Anerkennung bedeute. ${ }^{55}$ Das Auswärtige Amt in Bonn war ähnlicher Meinung. ${ }^{56}$ Was der israelischen Öffentlichkeit weiter vorenthalten wurde, war längst offenkundig: Israel und die Bundesrepublik pflegten Kontakte im öffentlichen, wirtschaftlichen, politischen und schließlich auch im gesellschaftlichen Bereich. Bald sollten auch militärische und halbmilitärische Verbindungen folgen. Die Rolle Israels, des Nahen Ostens und der Bundesrepublik im internationalen Kräftespiel war bereits scharf umrissen. Bonn handelte nicht nur aus eigener Initiative. Die Kritik an der Bundesrepublik verfehlte deshalb manchmal den Adressaten.

Das Schilumimabkommen stellte gleichsam eine weitere Verbindung zwischen der Bundesrepublik und den Alliierten her, denen die moralischen und propagandistischen Vorteile der Zusammenarbeit zwischen dem Staat Israel und der Bundesrepublik Deutschland auf dem Höhepunkt des Kalten Krieges nicht entgangen waren. Der Umstand, daß sich der ehemalige Verfolger und das ehemalige Opfer zusammen im westlichen Lager wiedergefunden hatten, veranschaulichte die Bedeutung gemeinsamer demokratischer Werte angesichts der kommunistischen Herausforderung und Bedrohung. Die Gesten der Bundesrepublik gegenüber Israel waren in gewisser Hinsicht der Preis, den sie für den militärischen Schutzschirm gegen die Gefahr aus dem Osten bezahlen mußte, und ein weiteres klares Bekenntnis zu liberalen, humanistischen, demokratischen Grundwerten, deren Erhaltung der Westen auf seine Fahnen geschrieben hatte. Durch die Annäherung an Israel verband die Bundesrepublik die Bekräftigung des Vertrauens in die westlichen Werte mit der Distanzierung von der belastenden Vergangenheit. Auch im verbalen Schlagabtausch mit der DDR über die Wiedervereinigung und über den politischen Geist der westlichen Erbin des Dritten Reiches kam der Legitimierung der Bundesrepublik durch die jüdische Seite entscheidende moralische Bedeutung zu.

Das Abkommen mit Israel besaß schließlich auch deshalb großes Gewicht für die Bundesrepublik, weil es zur Verbesserung des Ansehens Westdeutschlands in der amerikanisch-jüdischen Gemeinschaft und in der breiten amerikanischen Öffentlichkeit behilflich sein konnte. Nicht zuletzt Adenauer maß dem amerikanischen Judentum beträchtlichen Einfluß auf die Meinungsbildung in der amerikanischen Gesellschaft bei. Daher rührten u.a. die erheblichen Anstrengungen westdeutscher Diplomaten, auf die Bitten und Wünsche der amerikanischen Juden einzugehen. ${ }^{57}$

55 SHINNAR, Bericht eines Beauftragten, S. 68.

56 Vermerk von Dr. Voigt vom 16. 6. 1955, PA, III, 316-82.00.

57 JelineK, Political Acumen, S. 81-82; SHAFIR, Postwar Getman Diplomats, S. 155-201. 
Die Publizität rund um die Schilumim sowie weitere Kontakte zwischen Israel und der Bundesrepublik waren auch der israelischen Regierung ein Anliegen. Die Regierung stand unter dem Druck der Öffentlichkeit, die, wie man annehmen darf, das Abstimmungsresultat in der Knesset mehrheitlich nicht billigte. Um einen Meinungsumschwung herbeizuführen, setzte die israelische Regierung nicht nur auf den positiven Effekt des Schilumimgüterflusses und der individuellen Entschädigung, sondern Vertreter des Außenministeriums stellten auch Überlegungen an, wie man die öffentliche Meinung beeinflussen könne. Ein hochrangiger Diplomat machte den Vorschlag, eine Öffentlichkeitskampagne zugunsten der Bundesrepublik durchzuführen, um die öffentliche Meinung in Israel und anderswo vom guten Willen der Bundesregierung zu überzeugen. ${ }^{8}$ Teile der hohen Beamtenschaft im israelischen Außenministerium befürworteten die Annäherung, fürchteten jedoch den öffentlichen Protest.

Der israelische Durchschnittsbürger empfand tiefe Abscheu gegen die beiden deutschen Staaten und die Deutschen, mied jeglichen Kontakt mit Deutschland und zeigte wenig Verständnis für zukunftsorientierte Argumente, die dieser Anschauung widersprachen. Bei zahlreichen Israelis war der Schrecken des Holocaust noch lebendige Erinnerung. Von Deutschland wollten sie nichts wissen. Wer vor ungünstigen internationalen Entwicklungen, vor dem Kalten Krieg und dem zunehmenden internationalen Gewicht der Bundesrepublik warnte, blieb ein Rufer in der Wüste. Juden in Deutschland wie etwa Karl Marx und Hendrik van Dam hatten dagegen ein handfestes Interesse an der Verbesserung des Verhältnisses zwischen der Bundesrepublik und Israel. Diese beiden Persönlichkeiten taten das Ihre, um ehemalige deutsche Bürger jüdischer Herkunft sowie die israelische Elite von der Notwendigkeit einer Annäherung zwischen Israel und der Bundesrepublik zu überzeugen. Aber auch die Überreste des deutschen Judentums in Israel, in den Vereinigten Staaten und in Großbritannien leisteten einen Beitrag zur Verbesserung der Atmosphäre zwischen beiden Völkern. Dabei spielten materielle, gesellschaftliche und politische Interessen innerhalb der jüdischen Gemeinschaft eine Rolle, aber auch irrationale Motive, wie Reste von deutschem Patriotismus, verklärte Erinnerungen an deutsche Städten und Landschaften und nostalgische Sehnsüchte an die deutsche Kultur und Sprache.

Jüdische Funktionäre in den USA, in Großbritannien und in anderen Ländern sowie hochrangige israelische Diplomaten bewiesen in der Regel größeren Weitblick als der israelische Durchschnittsbürger. In ihren Briefen und Berichten kommt Besorgnis über ungünstige Entwicklungen der internationalen Politik, die wachsende internationale Bedeutung der Bundesrepublik, den zunehmenden Wettbewerb mit den arabischen Staaten um die Gunst der Bundesrepublik und über eine mögliche Wende in der deutschen Haltung gegenüber den Juden, Israel und dem Holocaust zum Ausdruck. Die Debatten im Außenministerium über deutsche Fragen waren emotionsgeladen. ${ }^{99}$ Ministerialdirektor Eytan faßte die

58 Yechiel Ilsar, stv. Abteilungsleiter für Westeuropa, an den Abteilungsleiter vom 20. 9. 1954, ISA, 3309/25.

59 Ruth Wolff an Eytan vom 26. 4. und 13. 5. 1953; Citroen an Eytan vom 4. 5. 1953, Citroen an Leo Kohn vom 11. 5. 1953, ISA, 2413/2. 
vorherrschende Haltung der israelischen Diplomatie wie folgt zusammen: „Bevor wir zu laufen beginnen, müssen wir gehen lernen." ${ }^{60}$ Bis sich die israelische Öffentlichkeit mit einem deutschen Gesandten abfinde, sei es noch ein weiter Weg, selbst wenn die deutsche Vertretung in Jerusalem errichtet werde. ${ }^{61}$ Die Öffentlichkeit müsse zuerst gründlich darauf vorbereitet werden, so der Ministerialdirektor. Eytans Äußerungen sind als Hinweis darauf zu werten, daß einige hochrangige Vertreter des Ministeriums Beziehungen mit der Bundesrepublik Deutschland auf längere Sicht befürworteten. Führende israelische Politiker und jüdische Funktionäre, darunter Ben Gurion, Sharett und Goldmann unterstützen diese Haltung in der einen oder anderen Form, ganz im Gegensatz zur kategorischen Ablehnung jeder Öffnung gegenüber Deutschland in der öffentlichen Meinung, getragen von den dominanten Massenblättern und einer Handvoll seriöser Zeitungen sowie von politischen Extremisten und einer Reihe von Organisationen von Holocaust-Überlebenden, aber auch von einem Großteil des politischen Establishments. Dieses war aber überwiegend pragmatisch eingestellt, somit theoretisch leicht umzustimmen. Die Leitung des israelischen Außenministeriums vertrat dennoch in den folgenden zwei Jahren bis 1955 die Auffassung, daß die israelische Öffentlichkeit "noch nicht reif“ sei für eine drastische Verbesserung der Beziehungen zum deutschen Staat.

\section{Israel denkt um - Die Frühphase}

Die Aufnahme diplomatischer Beziehungen zu Israel war für die Bundesrepublik ähnlich wie die Schilumim ein symbolischer Akt der „Rückkehr zur Völkerfamilie". Zusätzlich wurde sie als moralischer Schlußstein eines materiellen Abkommens und als Zeichen der Würdigung der westdeutschen Hilfsbereitschaft für den jungen jüdischen Staat gewertet. Dieses Leitmotiv der „Hilfe für den jüdischen Staat" sollte später mehrmals wieder auftauchen. Sehr häufig wurde die Frage gestellt, weshalb die Bundesregierung nach Inkrafttreten des Schilumimabkommens noch auf die zahlreichen israelischen Forderungen eingehe. Man habe doch schließlich guten Willen demonstriert, materielle Hilfe geleistet und damit die Verbrechen der Nationalsozialisten wiedergutgemacht. Die „ewigen israelischen Forderungen" wurden als ermüdend, unfair und manchmal auch als böswillig gewertet. Solchen Klagen gegen die „unvernünftigen Israelis“ hafteten oft Rassismus und Stereotypisierung an. Doch auch israelische und jüdische Vertreter wurden nicht müde, die Reue Deutschlands gegenüber der jüdischen Gemeinschaft und dem Staat Israel anzumahnen, bis dieses Argument zum demagogischen Spielball skrupelloser israelischer Politiker verkam, die sich nicht scheuten, das Thema „Endlösung“ für alltägliche politische Zwecke zu mißbrauchen. Das israelische Nein zu diplomatischen Beziehungen mit der Bundesrepublik wurde in Deutschland als Affront empfunden. Bundeskanzler Adenauer, im vollen Bewußtsein der

60 Eytan an Shinnar vom 22. 7. 1953, ISA, 572/2a.

61 Ebd., dort Verweis auf die Auseinandersetzung über den Status von Jerusalem. 
Erwartungen und Gefühle seiner Landsleute, versuchte diesen Eindruck zu dämpfen, indem er am 4. März 1953 bei seiner Eröffnungsrede zur Debatte über das Schilumimabkommen im Bundstag für Geduld plädierte:

„Wir haben daher die berechtigte Hoffnung, daß der Abschluß dieser Verträge schließlich auch zu einem ganz neuen Verhältnis zwischen dem deutschen und dem jüdischen Volke wie auch zu einer Normalisierung der Beziehungen zwischen der Bundesrepublik und dem Staate Israel führen wird. Wir werden hierbei nach allem, was vorgefallen ist, Geduld zeigen und auf die Auswirkung unserer Wiedergutmachungsbereitschaft und schließlich auf die heilende Kraft der Zeit vertrauen müssen. ${ }^{462}$

Solche Appelle sollten sich in den folgenden Monaten in der einen oder anderen Form wiederholen, bis die Pläne zur Aufnahme diplomatischer Beziehungen zwischen beiden Ländern endgültig zurückgestellt wurden.

Das Ausbleiben formaler Beziehungen verursachte ein gewisses Unbehagen, vor allem auf deutscher Seite. Die Israelis hatten ihren "Horchposten“ in Köln, einschließlich konsularischer Vertretung. Israelische Journalisten konnten sich in der Bundesrepublik frei bewegen, und viele Israelis beherrschten die deutsche Sprache. Ganz anders verhielt es sich für die Bundesrepublik: Das britische Konsulat in Haifa, bzw. ein einziger Beamter dieses Konsulats, nahm bis 1965 gewisse konsularische Aufgaben für die Bundesrepublik wahr, obwohl London, besonders nach Inkrafttreten des Deutschlandvertrags im Mai 1955, gerne auf diese Stellvertreterfunktion verzichtet hätte. ${ }^{63}$ Die israelischen Behörden waren bei der Erteilung von Einreisevisa für deutsche Bürger, einschließlich Journalisten usw. lange Zeit äußerst zurückhaltend. Jede Ausnahme von dieser Regel wurde gebührend vermerkt. Vor der Niederlassung Rudolf Küstermeiers und seiner jüdischen Ehefrau in Israel verfügte nicht einmal die deutsche Nachrichtenagentur dpa über einen Reporter im Land. Regierungssprecher und Staatssekretär von Eckardt erachtete die aktuelle und objektive Berichterstattung aus Israel als unerläßlich und beauftragte damit seinen persönlichen Freund, den Arzt Dr. Martin Hirsch aus Jerusalem (später Berlin). Bonn suchte und fand zusätzliche Informationsquellen. Aber selbst eine so einfache Dienstleistung wie Übersetzungen aus dem modernen Hebräisch bereitete in den fünfziger und sechziger Jahren in Bonn Schwierigkeiten. Auch die Versuche, Israel aus Ostjerusalem und Amman zu beobachten, und die unausgewogenen Briefe von in Israel niedergelassenen deutschen Missionaren konnten die Lücke nicht füllen. Die nicht sehr erfolgreichen Versuche des Auswärtigen Amts, verläßliche Information über Israel zu beschaffen, veranschaulichten das Problem der fehlenden Beziehungen.

In Israel fielen die politischen Angelegenheiten in Zusammenhang mit Deutschland in den Zuständigkeitsbereich des Außenministeriums. Das Finanzministerium betreute die wirtschaftlichen Fragen. Die Jewish Agency for Palestine (JAFP) und der Jüdische Weltkongreß (WJC) hatten als Brückenbauer des deutsch-israelischen Dialogs seit der Unterzeichnung des Luxemburger Abkom-

62 Verhandlungen des Deutschen Bundestages, 1. WP., Stenographische Berichte, Bd. 15, S. 12.095 .

${ }^{63}$ Die britische Botschaft in Tel Aviv an die Abteilung Levante vom 15. 5. 1955; Notiz von C.G. Marp an die West-Abteilung vom 9. 6. 1955, PRO, FO 371/115819, VR 10318/1. 
mens keine offizielle Funktion mehr, mit Ausnahme von Nahum Goldmann als Präsident der Claims Conference und des WJC. Goldmann traf sich regelmäßig mit Bundeskanzler Adenauer und anderen führenden deutschen Persönlichkeiten, manchmal trotz öffentlicher oder diskreter israelischer Kritik. In punkto Weltanschauung galt der WJC-Präsident als Mann des Ausgleichs. Er distanzierte sich von gewissen militanten Standpunkten Ben Gurions und dessen Umgebung und geriet deshalb manchmal in der israelischen Boulevardpresse unter Beschuß. Einige Aspekte seines Verhaltens waren umstritten, was die Israelis zuweilen veranlaßte, ihm Information vorzuenthalten, um sein Eingreifen zu verhindern. Goldmanns Fähigkeiten, seine Verbindungen zu führenden Staatsmännern, seine Neugier und sein Tatendrang machten ihn jedoch zum unverzichtbaren Teilnehmer sowohl der offenen als auch der geheimen Aktivitäten des Staates Israel, einschließlich der im Zusammenhang mit den diplomatischen Beziehungen, den Verhandlungen über Anleihen und finanzielle Hilfe an Israel, den Rüstungsgeschäften und weiteren deutsch-israelischen Angelegenheiten. Wo andere in Bonn scheiterten, hatte Goldmann Erfolg. Ben Gurion, Sharett und Meir waren trotz Antipathie, Mißtrauen und Befürchtungen auf Goldmanns Dienste angewiesen. Jeder einzelne Aspekt der Beziehungen zwischen der Bundesrepublik Deutschland und Israel verriet seine Spur. ${ }^{64}$

In seiner zweiten Amtsperiode als Ministerpräsident gestaltete und leitete Ben Gurion die israelische Politik gegenüber der Bundesrepublik in vorderster Front, nachdem er diesbezüglich vorher in Sharetts Schatten gestanden hatte, solange jener für die auswärtigen Angelegenheiten Israels zuständig war. Sharett hatte bis zu seinem Rücktritt im Frühjahr 1956 seine ganze Autorität für die Normalisierung des Verhältnisses zu Deutschland in die Waagschale geworfen und war dabei von Ministerialdirektor Eytan, Yachil, Shinnar und anderen Deutschlandexperten unterstützt worden. Ein Großteil der Beamten des israelischen Außenministeriums erachtete die Annäherung an die Bundesrepublik als unumgänglich. Zur Begründung wiesen die aus Mitteleuropa (Deutschland, Österreich, Tschechoslowakei) stammenden Beamten auf den Wandel in der öffentlichen Meinung in Westdeutschland, auf die sich verändernde Rolle der Bundesrepublik in Europa und im westlichen Bündnissystem sowie auf den wachsenden Einfluß der arabischen Staaten hin. Die Bedeutung und die Rolle der Emotionen wurde nicht bestritten, doch die Realpolitik, das heißt die unmittelbaren und zukünftigen Bedürfnisse des Staates, erhielten den Vorrang. Darauf galt es, wie gesagt, die Öffentlichkeit vorzubereiten. Ben Gurions Comeback nach Sharetts Rücktritt verlieh der israelischen Deutschlandpolitik pragmatischere Züge. Statt diplomatische Erfolge waren nun vor allem praktische bzw. materielle Lösungen gefragt.

Adenauer traf sich sporadisch mit Goldmann und Shinnar und war mit der Lage in Israel offensichtlich bestens vertraut. Während er Ende 1953 noch auf den Austausch von Diplomaten drängte, ließ sein Druck aufgrund einer realistischen Einschätzung der Lage in Israel später nach. Regierungssprecher von Eckardt soll einem israelischen Journalisten gegenüber am 17. Dezember 1954, rund fünf

64 SHAFIR, Goldmann ve Adenauer, S. 59-83. 
Monate vor Inkrafttreten des Deutschlandvertrages, gesagt haben: „Die Entscheidung über den Zeitpunkt der Aufnahme diplomatischer Beziehungen bleibt Ihnen [Ihrem Staat] überlassen. Sie sollte aber eine beträchtliche Mehrheit in der Knesset finden." 65 Yachil bezweifelte die Authentizität dieser Aussage. Zahlreiche israelische Politiker und Journalisten wiesen auf das vermeintlich starke Verlangen der Bundesrepublik nach Normalisierung des Verhältnisses mit Israel hin und leiteten daraus ab, daß sich Israel Zeit lassen könne. Lautstarke Forderungen in der westdeutschen Presse in diesem Sinne verstärkten diesen Eindruck noch zusätzlich. ${ }^{66}$ Am 14. September 1953 forderte Adenauer im Gespräch mit der Zeitung Die Welt die Normalisierung der Beziehungen mit Israel. Ein Jahr später, wieder in Die Welt, kam der Kanzler auf diese Forderung zurück. Israelische Diplomaten bemerkten verärgert: Adenauer versucht uns zur Normalisierung zu zwingen. Doch war Adenauer zu jenem Zeitpunkt tatsächlich an der Normalisierung interessiert? Zwei Monate später machte Hallstein gegenüber der Frankfurter Allgemeine Zeitung eine ähnliche Aussage, die sich als pures Ablenkungsmanöver entpuppte. Angesichts der weltpolitischen Entwicklungen und des gestiegenen Ansehens der Bundesrepublik im Westen dürfte der Staatssekretär dem Staate Israel nur noch abnehmende Bedeutung beigemessen haben. ${ }^{67}$ Man darf also auch Adenauers Aufrichtigkeit in dieser Sache bezweifeln. Seine Aufforderungen an Israel klangen nicht besonders eindringlich.

Im Rückblick betrachtet, fällt die Doppelstrategie gegenüber Israel auf, die der Bundeskanzler während seiner gesamten Amtszeit bis zu seinem Rücktritt im Jahre 1963 betrieben hat. Mit fast regelmäßiger Häufigkeit stellte er die Aufnahme diplomatischer Beziehungen zu Israel in Aussicht, worauf er jeweils eine Erklärung folgen ließ, weshalb dies zum gegebenen Zeitpunkt nicht möglich sei. Nicht viel anders agierte Heinrich von Brentano. Als Geste des guten Willens benachrichtigte er im Juni 1955, nachdem er zum Bundesaußenminister ernannt worden war, Shinnar zwar persönlich über seine Ernennung und erweckte damit wohl einige Hoffnungen bei dem Leiter der israelischen Einkaufsmission, ${ }^{68}$ die auch nicht unberechtigt erschienen. Denn Brentano hatte sich schon als Fraktionsvorsitzender im September 1952 grundsätzlich für Warenlieferungen an Israel ausgesprochen. Er hatte damals aber auch auf das Problem „der Rückwirkungen in der arabischen Welt" hingewiesen. ${ }^{69}$ Die hierin zum Ausdruck kommende Reserve gegenüber dem Dialog mit Israel ist offensichtlich nach seinem Amtsantritt als Außenminister nicht zuletzt auf Anraten der zuständigen Beamten im Auswärtigen Amt verstärkt worden, wie seine zögerliche Gangart und die Beratungen mit dem Kanzler dann zeigten. Solange von Brentano im Amt war, gab es also wenig

65 Yachil an Eytan vom 17. 12. 1954, ISA, 2413/3.

66 StUtTgarter Zeitung vom 25. 9. 1953; FrankfurTer Allgemeine Zeitung vom 30. 9. 1953 und 8. 11. 1954; RheINISCHE POST vom 2. 10. 1953 und 3. 10. 1953; Die WELT vom 14. 9. 1954.

67 Ausarbeitung „Außenpolitische Lage der Bundesrepublik“ vom 8. 11. 1954, PA, 210.00, Bd. 36.

68 Shinnar an Ilsar vom 6. 5. 1955, ISA, 613/7; Shinnar an Ilsar vom 5. 6. 1956, ISA, 3099/25.

69 KABINETTSPROTOKOLLE, 1952, 245. Kabinettsitzung am 8. 9. 1952, S. 557. 
Hoffnung für eine Verbesserung des Verhältnisses zwischen der Bundesrepublik Deutschland und dem Staat Israel..$^{70}$ Die Israelis ihrerseits glaubten, daß der Fortschritt in diesem Bereich allein von ihnen abhängen würde.

\section{Bonns Widerstand gegen diplomatische Beziehungen $\mathrm{zu}$ Israel}

Nachdem es den arabischen Staaten nicht gelungen war, die Ratifikation des Schilumimabkommens zu verhindern, nahmen Häufigkeit und Intensität der arabischen Interventionsversuche im deutsch-israelischen Verhältnis ab April 1953 deutlich zu. Davon versuchte auch die DDR zu profitieren, nachdem die Sowjetunion zur Einsicht gelangt war, daß sich die. Einmischung im Nahostkonflikt lohnen könnte. Die arabischen Staaten waren ihrerseits bestrebt, die Unterstützung der längst nicht mehr als westliche Domäne geltenden Entwicklungsländer zu gewinnen. Der Regierungswechsel in Washington bzw. die Wahl des Republikaners Dwight D. Eisenhower zum amerikanischen Präsidenten im November 1952 entpuppte sich zudem als schwerer Schlag für Israel. In der Hoffnung auf arabische Unterstützung bei der Eindämmung des Kommunismus verfolgten Präsident Eisenhower und sein Außenminister John Foster Dulles eine neutrale Politik im Hinblick auf Israel und seine arabischen Nachbarn ${ }^{71}$, die sich oft zu Israels Nachteil auswirkte. ${ }^{72}$ Westdeutschland wurde unversehens zum weiteren Nebenschauplatz des Nahostkonflikts. Je mehr die Bundesrepublik in die internationale Staatengemeinschaft integriert war, desto weniger war sie auf Israel angewiesen und desto mehr büßten jüdische Angelegenheiten in ihren Augen an Dringlichkeit ein. Moralische und ethische Fragen sowie Emotionen traten in den Hintergrund. Auch die Eisenhower-Regierung schenkte deutsch-israelischen Fragen nur wenig Beachtung. Bonn gegenüber konnte sich die israelische Regierung somit nur noch auf sich selbst verlassen sowie auf die Hilfe der jüdischen Gemeinden in den USA und in Großbritannien. ${ }^{73}$

Gleichzeitig verschlechterte sich Israels Verhältnis zum Ostblock. Der Sowjetunion war nicht entgangen, daß Israel die Bundesrepublik (gegenüber der DDR) bevorzugte und sich damit stärker an den - antikommunistischen - Westen band. Im Zuge der Einbindung der DDR in den Ostblock als Reaktion auf entsprechende Entwicklungen im Westen griff man zu den Winkelzügen der Dialektik und schob Israel die Schuld an der Verschlechterung der Beziehungen zu: Der Osten verstärkte seine Angriffe auf Israel. Dem jüdischen Staat wurde Kompli-

70 Der First Secretary Raymond E. Lisle, US-Botschaft in Bonn, an den Außenminister, vom 5. 10. 1955, USNA, 662.84A/10-555.

71 Krekeler an Pawelke vom 19.3. 1953, IfZ-Archiv, ED 135/65; Niederschrift Krekelers über die Gespräche mit Byroade, Außenministerium am 6. 9. 1952, IfZ-Archiv, ED 135/ 63.

72 SPIEgEL, The other Arab-Israeli conflict; Altaras, Eisenhower and Israel.

73 SHafir, American Jews and Germany; SHAFIR, Der Jüdische Weltkongreß, S. 210-237; SHAFIR, Postwar German Diplomats, S. 155-201; TEMPEL, Legenden der Allmacht. 
zenschaft mit dem "neonazistischen, revisionistischen “ westdeutschen Staat vorgeworfen und darin ein „weiterer Beweis von Israels proimperalistischer Haltung" gesehen. Auch die jüdischen Gemeinden wurden in die antiisraelische Kampagne eingespannt. Zudem tadelten sämtliche Ostblockstaaten, allen voran die DDR, Israel wegen der Zusammenarbeit mit der Bundesrepublik. Nach dem Slánský-Prozeß und der Kritik an Israel kam es in der DDR zu Verfolgungen von jüdischen Bürgern und Sympathisanten, die sich für jüdische Bürger bzw. jüdische Anliegen einsetzten.

Die im Gefolge der Einbindung der Bundesrepublik in die westliche Staatengemeinschaft und der damit einhergehenden stärkeren Isolierung Israels gegebene neue Situation hatte unmittelbare Auswirkungen auf die Beziehungen zwischen der Bundesrepublik und dem Staat Israel. Während die israelische Seite ab 1956 damit beschäftigt war, das Umfeld für die Aufnahme diplomatischer Beziehungen vorzubereiten, versuchte das Auswärtige Amt, die Politiker von der Schädlichkeit eines solchen Schrittes zu überzeugen. Den Meinungsumschwung in Bonn haben wahrscheinlich die arabischen Staaten ausgelöst. Die Araber waren gegen jeden Schritt, der geeignet war, die internationale Stellung Israels zu verbessern und diesem Land damit zu helfen, die arabische Blockade zu durchbrechen und den arabischen Feindseligkeiten zu trotzen. Im Hinblick hierauf war es aus westdeutscher Sicht ein Vor- und kein Nachteil, daß Israel bis zu diesem Zeitpunkt der Aufnahme diplomatischer Beziehungen zur Bundesrepublik ablehnend gegenüber gestanden hatte. Diese Haltung befreite die Bundesregierung nicht nur von der Last, ihrerseits auf die Aufnahme diplomatischer Beziehungen zu drängen, sondern gab ihr außerdem die Chance, Israel die Verantwortung für die Nichtexistenz diplomatischer Beziehungen zuschieben zu können. Die Bundesrepublik sei nicht auf die Absolution der jüdischen Seite angewiesen, um in die Gemeinschaft der (antikommunistischen) Völker aufgenommen zu werden, hieß es in manchen Bonner Kreisen. Sollte Israel versuchen, das deutsche Volk bei seiner so hart erarbeiteten Wiederaufnahme in die internationale Gemeinschaft zu behindern oder zu erniedrigen, gebe es ja noch die Araber. Diese wüßten nämlich die freundschaftlichen Tätigkeiten des Auswärtigen Amts in arabischen und islamischen Staaten zu schätzen. ${ }^{74}$ Am Anfang gerieten die westdeutschen Vertreter in Verlegenheit, wenn ihre arabischen Partner über den "gemeinsamen Feind" und über den unlängst geführten gemeinsamen Kampf gegen einen gemeinsamen Feind sprachen. Doch für die Arabisten im Auswärtigen Amt waren solche Äußerungen nichts Neues. Nur wenige Dokumente enthalten freilich direkte Äußerungen in diesem Sinne. In den meisten Fällen ergeben sie sich aus der Entschlüsselung von Anspielungen und Andeutungen. ${ }^{75}$

74 HARF, Die Bedeutung der arabischen Staaten, S. 319-312.

75 Die US-Botschaft in Bagdad an das Außenministerium vom 28. 1. 1954, USNA, 662.87/ 1-2854; Presse- und Informationsdienst der Bundesregierung, dpa-Information betr. Politische Erfahrungen in Ägypten vom 25. 6. 1952; Aufzeichnungen Dr. Melchers vom 4. 3. 1952 und 6. 9. 1952, PA, III, 210-01/E, Bd. 1; Niederschrift üer die Besprechung beim Staatsseketrär vom 18. 9. 1952, PA, 210-01/E, Bd. 1; Aufzeichnung betr. die Deutsche Gesandtschaft in Jakarta vom 27. 9. 1952; Allandt an Blankenhorn, vom 9. 3. 1955, BArch, N 1351, Bd. 43; Kehrseite der Lieferungen an Israel. In: WilhelmSHAVENER ZeITUNG 
Arabische Vertreter aus dem öffentlichen und privaten Bereich beklagten sich bitter über die westdeutsche Politik gegenüber Israel. Sie warfen Bonn vor, die arabischen Interessen weitgehend zu mißachten und den Erzfeind der arabischen Nation privilegiert zu behandeln. Ohne Schilumim, so die arabische Argumentation, würde die israelische Wirtschaft und somit der Staat Israel zusammenbrechen. Vergeblich versuchten deutsche Vertreter wiederholt auf die moralischen Verpflichtungen der Bundesrepublik hinzuweisen. Nachdem es ihnen nicht gelungen war, das Schilumimprojekt als Ganzes zu Fall zu bringen, bissen sich die arabischen Staaten an Einzelheiten fest. So machten sie ihre deutschen Partner auf angebliche Verstöße Israels gegen einzelne Bestimmungen des Schilumimabkommens wie etwa das Wiederausfuhrverbot aufmerksam. Die Bundesregierung konnte diese Vorwürfe jedoch nicht bestätigen. Zur Verhinderung der Wiederausfuhr schlugen irakische Vertreter vor, die Schilumimgüter speziell zu kennzeichnen und der arabischen Seite eine Liste der gelieferten Güter sowie der nach Israel exportierenden Firmen zu überreichen, was von den westdeutschen Behörden jedoch abgelehnt wurde. Die geforderten Daten waren für die schwarzen Listen des Israel-Boykotts der Arabischen Liga bestimmt. ${ }^{76}$

Mit Argusaugen verfolgten die Araber sodann die Einhaltung des Lieferverbots für Militärgüter. Hier gab es massive Proteste und verschiedene Klagen über angebliche Lieferungen von Waffen und sogenannten strategischen Gütern, darunter Passagierschiffe und Fischerboote, die sich nach arabischer Darstellung leicht in Kriegsgerät verwandeln ließen. Die arabischen Staaten stellten Nachforschungen über westdeutsche Finanzhilfe für Israel an und protestierten gegen die Aufwertung des deutsch-israelischen Verhältnisses. Die Proteste gegen Beziehungen zwischen der Bundesrepublik und Israel führten dem Auswärtigen Amt die Brisanz des Themas für die Araber klar vor Augen. Syrien, Ägypten und Irak zeigten sich besonders aktiv, aber auch der Libanon, Saudi-Arabien und Jordanien blieben nicht untätig. Der ständige Strom von arabischen Klagen wurde von der westdeutschen Diplomatie als schwere Belastung empfunden. Deutsche Diplomaten in Bonn und im Ausland berichteten häufig über die schädlichen Auswirkungen des Schilumimabkommens auf die westdeutschen Interessen im Nahen Osten.77

Das Auswärtige Amt reagierte auf den arabischen Druck mit besorgter Zurückhaltung. Im Sommer 1955 berichtete Shinnar seinen Vorgesetzten über ein Nachlassen des deutschen Drängens auf diplomatische Beziehungen mit Israel und wies in diesem Zusammenhang besonders auf die zögerliche Haltung des Leiters der Länderabteilung im Auswärtigen Amt, des Gesandten Wolfgang Frhr. von Welck,

vom 8. 2. 1954; Zum Tage. In: RhEINISCHE POST (Düsseldorf) vom 20. 2. 1954; Mit spitzer Feder „Rommel-Rummel“. In: CellesCHe ZeItUNG vom 9.6. 1954.

76 Trützschler an Blankenhorn, Aufzeichnung, 9. 10. 1953; Vermerk betr. der Gesandte Saifullah Khandra vom 20. 5. 1954, PA, 210-01/E, Bd. 8; Aufzeichnung Froweins betr. der irakische Gesandte Saifullah Khandra vom 28. 5. 1954, B102/6419, Heft 1.

77 Von der Esch, Damaskus, an das AA vom 16. 10. 1955, PA, III, 210-01/E, Bd. 2; die Botschaft in Kairo an das AA vom 5. 8. 1955; Bericht von Dr. Melchers nach Bonn vom 15. 8. 1955; die Botschaft in Beirut an das AA vom 23. 8. 1955; die Botschaft in Djidda an das AA vom 29. 11. 1955, PA, 316, 81.00/1, 92.19; Pawelke an Krekeler vom 11. 11. 1952, IfZArchiv, ED 135/65. 
hin..$^{78}$ Etwa zur gleichen Zeit befragte das Auswärtige Amt die Vertretungen im Ausland nach ihrer Meinung zur möglichen Aufnahme diplomatischer Beziehungen zu Israel.

$\mathrm{Da}$ die israelische Diplomatie die Situation falsch beurteilte, behandelte sie das Problem mit einer Doppelstrategie. Einerseits rechtfertigte sie nach wie vor die Nichtaufnahme von diplomatischen Beziehungen mit der Bundesrepublik bei gleichzeitigen Bemühungen, das westdeutsche Interesse an Israel wachzuhalten. Andererseits war sie unvermindert damit beschäftigt, das politische Establishment in Israel - besonders Mapai-Vertreter - und die Öffentlichkeit von der Notwendigkeit diplomatischer Beziehungen mit Bonn zu überzeugen. Wie bereits gezeigt wurde, führte diese Überzeugungsarbeit bei Ben Gurion, Sharett, Josephthal, Peretz Naphtali und einer Reihe weiterer Regierungsvertreter und Knessetmitglieder schon früh zum Erfolg. Bemerkenswert ist der Fall des Erziehungsministers Zalman Aranne, der die Bundesrepublik am 28. September 1955 als einzige Großmacht bezeichnete, die aus echter Freundschaft mit Israel ohne irgendwelche Nebenabsichten handle, nachdem er sich kurz zuvor noch gegen das Einlaufen deutscher Schiffe mit gehißter deutscher Flagge in israelische Häfen ausgesprochen und vor Demonstrationen gegen die Anwesenheit deutscher Seeleute gewarnt hatte. ${ }^{j 9}$ Aranne forderte die Bundesrepublik auf, sich zur Verteidigung des jüdischen Staates zu bekennen. Nur so werde das deutsche Volk von seinen Sünden gegen die Juden befreit. 80

Die wachsende Zahl israelischer Korrespondenten in der Bundesrepublik gab den deutschlandfreundlichen Stimmen in der israelischen Tagespresse Auftrieb, und auch die im Hafen von Haifa gelöschten Schilumimgüter trugen das Ihre zur Schaffung eines freundlicheren Klimas bei. 1955 reisten die ersten zuvor von den israelischen Behörden auf ihre Vergangenheit überprüften deutschen Touristen in Israel ein. Gleichzeitig wurden auch die ersten offiziellen Gäste aus Deutschland empfangen. Die ehemaligen Wassenaar-Unterhändler Franz Böhm und Abraham Frowein besuchten Israel auf Einladung der Schilumimgesellschaft. Böhms Besuch fand im März 1954 statt, derjenige von Frowein im September 1955. ${ }^{.1}$ Froweins Besuch bereitete dem israelischen Außenministerium etliches Kopfzerbrechen. Er mußte geheimgehalten werden. Der Gast aus Deutschland hatte über die bilateralen Beziehungen und die Besetzung der zukünftigen westdeutschen Vertretung in Israel Rede und Antwort zu stehen. Als besonders problematisch erwies sich die Ernennung des Leiters dieser Vertretung. Er mußte eine ungetrübte Vergangenheit aufweisen und gleichzeitig eine angesehene Persönlichkeit sein.

78 Shinnar an Ilsar vom 8. 7. 1955, ISA, 2516/4; Avner in London an Najar in Den Haag vom 12. 8. 1955, ISA, 2551/4.

79 SHARETT, Yomanim, Bd. 4, S. 1110, Eintrag vom 7. 8. 1955; Niederschrift über die Beratungen im Büro des Premierministers vom 26. 7. 1954, ISA, 2418/4.

80 SHARETT, Yomanim, Bd. 5, S. 1177.

81 A.R. Moore in Tel Aviv an P.S. Falla, Abteilung Levante vom 22. 3. 1954, PRO, FO 371/ 111063, VR 10318/2; Kanzler der britischen Botschaft in Tel Aviv an die Abteilung Levante vom 22. 10. 1955, PRO, FO 371/115819, VR 10318/2; Shinnar an Sharett vom 28. 9. 1955, ISA, 590/6; Aufzeichnung von Abt. 2, Frowein, vom 6.11. 1955, PA, $316,81.00 / 1$. 
Böhms Besuch in Israel erfolgte zu früh für konkrete Schritte in Richtung diplomatische Beziehungen. Trotzdem hat Böhm die israelische Entscheidung für die Aufnahme von Verhandlungen solcher Beziehungen im Herbst 1955 entscheidend mitbeeinflußt. Frowein reiste mit Erlaubnis seiner Vorgesetzten und wirkte als Überbringer inoffizieller Botschaften im beiderseitigen Auftrag. Seine Reise und der Besuch von Ministerialdirektor Janz im Frühjahr 1956 waren als Vorbereitung für die Aufnahme diplomatischer Beziehungen gedacht. ${ }^{82}$

\section{Das Scheitern der Normalisierung}

Zwei internationale Entwicklungen wurden in Israel als besondere Herausforderung empfunden: die Wiederbewaffnung Deutschlands und die Viermächtekonferenz in Berlin vom 25. Januar bis zum 18. Februar 1954. Die Wiederbewaffnung der deutschen Staaten hatte keine unmittelbare Auswirkungen auf Israel, doch sie rief, wie bereits mehrfach erwähnt, bittere Erinnerungen und allgegenwärtige Ängste wach. Die in erster Linie moralische und ethische Bedeutung dieses Schrittes machte ihn zum idealen Spielball rechter und linker Demagogen, so geschehen etwa auf einer Tagung des Jüdischen Weltkongresses im April 1954. Goldmann gelang es, die gemäßigteren Teilnehmer zu besänftigen und eine Kompromißresolution durchzusetzen, worin die Wiederbewaffnung verurteilt wurde, ohne Bonn oder den Westen zu brüskieren. ${ }^{83}$ Israel verurteilte ausdrücklich beide deutsche Staaten, offensichtlich mit der Absicht, den antiwestlichen Charakter dieser Kampagne aufzuweichen und um zu verhindern, daß die Sowjetunion von den antideutschen jüdischen Vorstößen profitierte. Da der Protest gegen die Wiederbewaffnung Deutschlands von prosowjetischen Elementen ausgegangen war, wurde die Resolution, die sich gegen beide Seiten richtete, als prowestlicher und Bonnfreundlicher Schritt empfunden. Die Knesset diskutierte am 15. November 1954 ausführlich die Wiederbewaffnung. Sämtliche Fraktionen verurteilten den Schritt. Die Rechte attackierte beide deutschen Staaten, die Linke vor allem die Bundesrepublik. ${ }^{84}$ In einer an alle Parlamente der Welt gerichteten Resolution brachte die Knesset im Namen der jüdischen Welt Besorgnis und tiefe Bestürzung über die Wiederbewaffnung beider deutscher Staaten zum Ausdruck und rief dazu auf, einen weiteren Holocaust zu verhindern. ${ }^{85}$ So grimmig die Formulierung war, sie vermied es, Bonn und den Westen direkt anzugreifen. Die Debatte und die anschließende Verurteilung spiegelten die wahren Gefühle der jüdischen Seite, gleichzeitig aber auch den Willen, Bonn mitten im Schilumimprogramm nicht vor

82 Shinnar an Sharett vom 28. 9. 1955, ISA, 590/6.

83 Resolution zur deutschen Wiederbewaffung, angenommen vom European Executive of the World Jewish Congress vom 29. 4. 1954; Protokolle der Versammlung der Londoner Vertreter der europäischen WJC-Exekutive vom 14. 10. 1954, CZA, Z 6/851; die Kanzlei der britischen Botschaft in Tel Aviv an die Abteilung Levante vom 23. 10. 1954, PRO, FO $371 / 10318 / 3$.

84 KNESSET-PROTOKOlle [Original hebr.], 2. Knesset, 496. und 497. Sitzung am 15./16. 11. 1954.

85 Die Resolution der Knesset vom 16. 11. 1954, BGA, Prime Minister's Office, Box No. 2. 
den Kopf zu stoßen. Das war jedenfalls die Essenz des Standpunktes von Goldmann und der linken Kritiker. Falls gewisse Vertreter im Auswärtigen Amt den jüdischen Staat noch einer neutralen Haltung zwischen Ost und West verdächtigten, die Knessetdebatte belehrte sie endgültig eines Besseren. ${ }^{86}$ Die Debatte legte aber auch die fortwährenden Ängste und den $\mathrm{Haß}$ gegen beide deutsche Staaten offen und deutete auf die lediglich begrenzte Unterstützung der israelischen Öffentlichkeit für die "Normalisierung" hin.

Die Viermächtekonferenz in Berlin rief in Israel besonderes Interesse hervor. Die israelische Regierung wünschte, als gleichberechtigte Delegation an dieser Konferenz teilzunehmen, sowie eine Verurteilung des Nationalsozialismus und des Holocaust in einem zukünftigen Friedensvertrag. Nach intensiven Beratungen im Außenministerium wurde beschlossen, Yachil als offiziellen israelischen Beobachter zur Konferenz zu schicken. Die israelische Regierung befürchtete, $\mathrm{da} ß$ sich ein vereintes Deutschland nicht an die von der Bundesrepublik eingegangenen Verpflichtungen gebunden fühlen könnte, und witterte Gefahr für das Luxemburger Abkommen. Da die DDR das Abkommen zuvor verurteilt hatte, war diese Möglichkeit nicht von der Hand zu weisen. Die israelische Regierung verhandelte mit den Großmächten und mit der Bundesrepublik und suchte das Gespräch mit der DDR, ohne Bonn darüber zu informieren. ${ }^{87}$ Während der Konferenzvorbereitungen verlangten die Israelis Klärung zu einigen Fragen, die Goldmann umgehend an Dulles herantrug. Der amerikanische Außenminister versprach, die Bundesrepublik werde ihren Verpflichtungen auch im Rahmen des zukünftigen Status Deutschlands nachkommen und Ostdeutschland dazu gezwungen werden, die westdeutschen Verpflichtungen zu respektieren.88

Als die Bundesrepublik am 5. Mai 1955 die Souveränität erlangte, betraf dies zumindest formal auch die bilateralen Beziehungen zwischen der Bundesrepublik und Israel. Die britische Regierung reagierte darauf mit der Überprüfung des Status der konsularischen Dienste für die Bundesrepublik im britischen Konsulat in Haifa. Bereits am 3. Februar hatte Shinnar das Außenministerium in Jerusalem über ein Gespräch zu dieser Frage im Auswärtigen Amt informiert. Da das britische Konsulat in Haifa die Stellvertretung für die Bundesrepublik einzustellen beabsichtigte, schlug das Auswärtige Amt vor, bis zur endgültigen Klärung der Frage vorläufig einen Beamten zur Ausstellung von Sichtvermerken nach Israel zu entsenden. ${ }^{89}$ Shinnar versprach seinen deutschen Gesprächspartnern, die Angelegenheit bei seinem nächsten Besuch in Israel im Juni zur Sprache zu bringen und fügte hinzu, daß ein solcher Beamter sich auch um die Verwaltung der individuellen Entschädigung kümmern könnte. Sharett nahm Shinnars Bericht zur Kenntnis und beschloß, die Antwort aufzuschieben. Der israelische Außenminister hatte das Gefühl, daß sich Israel früher als ursprünglich erwartet über das Verhältnis zu Deutschland würde entscheiden müssen, und erachtete eine vorübergehende

86 Yachil an die Abteilung für Westeuropa und Öffentlichkeitsarbeit vom 8. 10. 1954, ISA, 2527/12; STUTTGARTER ZEITUNG vom 20. 9. 1954.

87 Livneh an die Abteilung Osteuropa vom 25. 3. 1953, ISA, 2511/17a.

88 John F. Dulles an Nahum Goldmann vom 2. 2. 1954, ISA, 419/11.

89 Shinnar an Najar vom 3. 2. 1955, ISA, 605/2. 
Lösung als zu unberechenbar. ${ }^{90}$ Das Auswärtige Amt tat also offensichtlich den ersten Schritt, und die israelische Regierung ließ diese Gelegenheit ungenutzt verstreichen.

Das Jahr 1955 war eines der schwierigsten in der Geschichte des jüdischen Staates. An seinen Grenzen wurde weiter gekämpft, und Freischärler aus Ägypten, Syrien und Jordanien, sogenannte Fedajin, verübten blutige Anschläge auf die israelische Zivilbevölkerung. Die Bevölkerung reagierte mit zunehmender Verbitterung, die sich auch im Militär breitmachte, und Ministerpräsident Sharett, ein friedfertiger Mann, der den Ausgleich mit den arabischen Nachbarn suchte, konnte dem Druck der Streitkräfte nicht mehr standhalten. Nach einem besonders barbarischen Anschlag auf israelische Zivilisten sah er sich gezwungen, einen begrenzten Vergeltungsschlag zu genehmigen. Doch statt zu einer begrenzten Aktion kam es am 1. März 1955 zu einem Großangriff auf eine ägyptische Militärbasis im Gazastreifen, bei dem 37 Ägypter und 9 Israelis ums Leben kamen. ${ }^{91}$ Israel wurde darauf hin am 30. März vom UNO-Sicherheitsrat einseitig verurteilt. Die Resolution kam, wie so oft bei antiisraelischen Beschlüssen dieses Gremiums, durch eine Koalition des Ostblocks und der blockfreien Staaten zustande, während sich der Westen einmal mehr abseits hielt, um die „Blockfreien“ nicht zu verärgern. Die israelische Öffentlichkeit wurde gegen den „unparteiischen" Schiedsrichter aufgehetzt, und die militanten Stimmen gewannen die Oberhand. Einem Aufruf folgend, kehrte Ben Gurion 1955 in das Amt des Ministerpräsidenten zurück, nachdem es ihm gelungen war, Sharett aus dieser Position zu verdrängen.

Das Auswärtige Amt nutzte das Mißgeschick der Israelis, indem es zu ihnen deutlich auf Distanz ging. Hallstein sagte im April 1956 vor deutschen Diplomaten, daß er die Verurteilung Israels durch den UNO-Sicherheitsrat nach dem Zwischenfall in Gaza zum Anlaß genommen habe, ein israelisches Kreditgesuch abzulehnen, dessen Bewilligung die Araber verärgert hätte. ${ }^{92}$ Das Auswärtige Amt, aber auch Adenauer und von Brentano, machten es sich zur Routine, arabisch-israelische Spannungen als Vorwand zur Ablehnung israelischer Gesuche zu nutzen. Um die Spannungen nicht zusätzlich zu erhöhen, wolle man dem israelischen Gesuch zur Zeit nicht entsprechen, so lautete die abschlägige Antwort zumeist.

Die israelische Regierung versäumte die Gelegenheit zur Aufnahme diplomatischer Beziehungen mit der Bundesrepublik im Mai 1955 unmittelbar vor Inkrafttreten des Deutschlandvertrages und ein weiteres Mal, etwa zwei Monate später, vor dem Dreimächtegipfel in Genf zwischen dem 18. und 23. Juli, der der deutschen Wiedervereinigung gewidmet war und an dem auch Beobachter beider deutscher Staaten teilnahmen. Shinnar zufolge hätte Israel bei dieser Gelegenheit Bedingungen aushandeln können, die auch für die Opposition im eigenen Land akzeptabel gewesen wären, und dabei vom Willen westdeutscher Politiker profitieren können, das internationale Ansehen der Bundesrepublik noch vor der Er-

90 Ilsar an Shinnar vom 22. 2. 1955, ISA, 605/2.

91 SHAhAM, Israel, S. 116; SHARETT, Yomanim, Eintrag vom 30. 5. 1955, S. 878.

92 Aufzeichnung Staatssekträr Hallsteins vom 3.-7. 4. 1956, PA, BSTS, Bd. 340. 
langung der Souveränität zu heben. ${ }^{93}$ Die israelische Opposition gegen Beziehungen zu Deutschland blieb zunächst unverändert stark, doch allmählich mehrten sich die Stimmen der Befürworter und Warnungen vor den Folgen einer weiteren Verzögerung dieser Angelegenheit. Die Mapai-Abendzeitung HaDor charakterisierte den sich abzeichnenden Stimmungswandel als „Allianz mit dem Feind von gestern gegen den Feind von heute“. Mit dem „Feind von gestern" war Deutschland und mit dem "Feind von heute" die Sowjetunion gemeint. ${ }^{94}$ Doch zur Enttäuschung vieler bewirkte der neue Status der Bundesrepublik keine Veränderung des offiziellen deutsch-israelischen Verhältnisses. Das amerikanische Außenministerium, das auf einen Wandel im deutsch-israelischen Verhältnis gehofft hatte, wies die Botschaft in Tel Aviv an, „die Israelis auf geeignete Weise informell darauf hinzuweisen, daß eine Sichtvermerkstelle der BRD nicht unbedingt eine israelische Anerkennung der Bundesrepublik bedeutet". Washington wollte mindestens eine deutsche Sichtvermerkstelle in Israel. 95

Israel reagierte ähnlich auf die Berliner Konferenz und den Genfer Gipfel. Aus Furcht vor ungünstigen Beschlüssen versuchte die israelische Regierung mit den Teilnehmern einzeln zu verhandeln. Man wundert sich, daß Jerusalem nicht versucht hat, das Problem durch die Aufnahme diplomatischer Beziehungen endgültig zu lösen und damit die Großmächte und die DDR vor vollendete Tatsachen zu stellen. Ein solcher Schritt hätte die Großmächte schlagartig ihrer Vermittlerrolle und die Ostdeutschen ihrer Erpresserposition beraubt. Auch den Arabern wäre keine Gelegenheit mehr geboten gewesen, die Bundesrepublik mit Drohungen unter Druck zu setzen. Doch anstatt den Gordischen Knoten zu durchtrennen, verschwendete die israelische Regierung wertvolle Zeit mit Nebenverhandlungen und spielte damit den deutschen (und arabischen) Gegnern der Normalisierung in die Hände. 96

Shinnar berichtete seinen Vorgesetzten im Juli 1955, wie erwähnt, daß das Auswärtige Amt nicht mehr die Aufnahme diplomatischer Beziehungen, sondern nur noch israelisches Entgegenkommen in bestimmten Teilbereichen fordere. Die israelische Regierung kam diesen Wünschen in der Regel nach: So unterstützte das israelische Außenministerium fortan die Aufnahme der Bundesrepublik in internationale Organisationen und vergrößerte sowohl den Spielraum für Kontakte mit deutschen Diplomaten als auch die Zahl der Einreisebewilligungen für Bürger der Bundesrepublik. Die Beratungen im israelischen Außenministerium, der eine Reihe von Briefwechseln, Beratungen, Kabinettssitzungen und Gesprächen mit westdeutschen Persönlichkeiten wie Böhm und Spitzenvertretern der SPD vorausgingen, waren von der Empfehlung gekrönt, diplomatische Beziehungen mit der Bundesrepublik Deutschland aufzunehmen. Einige Vertreter forderten die so-

${ }^{93}$ Interview mit Shinnar. In: MA'ARIv (Tel Aviv) vom 25. 9. 1965.

94 So Shmuel Mikunis (Kommunisten) zum Haushaltsvorschlag: KNESSET-PrOTOKOLlE [Original hebr.], 2. Knesset, 681. Sitzung am 1.3.1955.

95 Das Außenministerium an die US-Botschaft in Tel Aviv vom 17.6. 1955, USNA, 321.9, Germany, RG84, Box 5.

96 Shinnar an Sharett vom 8. 2. 1955, ISA, 2539/4; Edward B. Lawson, US-Botschaft in Tel Aviv, an den Außenminister vom 7. 2. 1956, USNA, 611.84A/2-756. 
fortige Aufnahme voller diplomatischer Beziehungen, andere rieten, sich mit der Bundesrepublik zunächst auf die Einrichtung einer Handelsmission mit gewissen konsularischen Befugnissen zu verständigen. ${ }^{97}$

Die Forderung des Auswärtigen Amts nach Einrichtung einer westdeutschen Sichtvermerkstelle setzte den israelischen Entscheidungsprozeß in Gang. Sharett befürwortete allem Anschein nach eine Zweistufenlösung: zuerst eine Mission und dann volle diplomatische Beziehungen, worauf die offentliche Meinung in Israel freilich zuerst noch vorzubereiten sei. Die Verpflichtung seiner Regierung im Auge behaltend, wonach die Schilumim nicht zu diplomatischen Beziehungen führen dürfen, war Sharett allerdings streng darauf bedacht, die beiden Angelegenheiten voneinander zu trennen, was er auch deutschen Stellen gegenüber wiederholt betonte. ${ }^{98} \mathrm{Am} 8$. November 1955 notierte Sharett in sein Tagebuch, Shinnar sei nach Jerusalem gekommen, um Instruktionen hinsichtlich der Annäherung an Deutschland entgegenzunehmen. ${ }^{99}$ Einen Monat später, am 9. Dezember, schlug Shinnar dem Außenminister vor, von Brentano die Einrichtung einer deutschen Handelsmission in Israel anzubieten. ${ }^{100} \mathrm{Im}$ innenpolitischen Bereich gelang es Sharett, den Vorsitzenden der religiösen Mizrahi-Partei, Moshe Shapira, für diesen Standpunkt zu gewinnen. ${ }^{101}$ Mit Goldmanns Hilfe machte er sodann den Cherut-Führer Menachem Begin mundtod. Goldmann legte Begin geheime Briefwechsel über westdeutsche Militärhilfe an Israel vor. ${ }^{102} \mathrm{Ob}$ Goldmann ihn auch über die bevorstehenden Entwicklungen informiert hat, ist unklar. Böhm war über Sharetts Aufklärungsarbeit und über die Opposition gegen die Annäherung an Deutschland im Bilde. ${ }^{103}$ Sharett setzte sein ganzes politisches Prestige für die Normalisierung der Beziehungen zu Deutschland ein. Das Scheitern dieses Vorstoßes hat ihn zweifellos politisch geschwächt und möglicherweise im Juni 1956 dazu beigetragen, daß er auch als Außenminister zurücktrat, nachdem er im Jahr zuvor bereits das Amt des Ministerpräsidenten abgeben hatte.

Sharett hielt die Zustimmung der Bundesrepublik für gesichert. Doch dann überraschte Goldmann mit einer pessimistischen Einschätzung: Mitte Dezember 1955 meinte er Yachil gegenüber, die israelische Initiative komme eventuell schon zu spät. ${ }^{104}$ Dessen ungeachtet traf Shinnar am 22. Dezember von Brentano zum Gespräch. Die beiden diskutierten die weiteren Schritte der Annäherung im einzelnen sowie andere Angelegenheiten von beiderseitigem Belang. Shinnar erhielt

97 Niederschrift über die Beratungen im Büro des Generaldirektors am 13. 10. 1955; der stv. Abteilungsleiter für Westeuropa an das Ministerbüro vom 19. 10. 1955; der Politische Sekretär von Außenminister Zeev Scheck an Sharett vom 24. 10. 1955; der Rechtsberater Shabtai Rosenne an den Minister vom 24. 10. 1955, ISA, 2413/3b.

98 Harman an Ilsar vom 21. 10. 1955, ISA, 2413/3b; Memorandum vom 24. 10. 1955, ISA, $2539 / 4$.

99 ShareTt, Yomanim, Bd. 5, S. 1292.

100 Shinnar an Sharett vom 9. 12. 1955, ISA, 2413/3b.

101 SHARETT, Yomanim, Bd. 5, Eintrag vom 28. 12. 1955, S. 1318.

102 Die US-Botschaft in Tel Aviv an den Außenminister betr. Gebrauch von deutschen Wiedergutmachungsleistungen für israelische Verteidigungszwecke; hier: Besprechung mit Eliezer Shostak vom 10. 2. 1956, USNA, 611.84A/2-1056.

103 Böhm an Hallstein vom 19. 2. 1956, CZA, Z 6/2001.

104 Yachil an Eytan vom 16. 12. 1955, ISA, 2539/4. 
den Eindruck, daß von Brentano auch an einer gestuften Annäherung interessiert sei. ${ }^{105}$ Sharett betrachtete die Angelegenheit als reif, und am 15. Januar 1956 vertraute er seinem Tagebuch an: "In dieser Besprechung wurden die Beziehungen zur Bundesrepublik endlich genehmigt. ${ }^{{ }^{106}}$ Im Kabinett hatte er sich mit einer List beholfen: Er schlug die Aufnahme voller diplomatischer Beziehungen vor, obwohl er wußte, daß sein Vorschlag chancenlos war. Minister Shapira forderte als Kompromiß die Einrichtung einer westdeutschen Mission, und sein Vorschlag wurde mit zehn zu eins Stimmen bei vier Enthaltungen gebilligt. Sharett hatte sein Ziel erreicht. ${ }^{107}$ Das israelische Massenblatt Yedioth Achronoth druckte folgenden Kommentar: „Die zunehmende Isolierung des Staates, die sich entwickelnden Handelsbeziehungen zwischen beiden Staaten und die Waffengeschäfte zwischen arabischen und osteuropäischen Ländern erfordern engere Beziehungen zur Bundesrepublik." 108 Am 28. Januar 1956 kam es zu einer weiteren Besprechung zwischen Shinnar und von Brentano. Darin teilte von Brentano mit, daß er weiterhin an Beziehungen mit Israel interessiert sei und arabische Interventionen nicht zulassen werde. Da jedoch etliche seiner Beamten befürchteten, die Araber könnten die sowjetische Besatzungszone anerkennen, wolle er - wie Shinnar berichtete deshalb zuerst die westlichen Alliierten konsultieren. Die beiden Seiten tauschten zum ersten Mal die Rolle. Shinnar versuchte den Bundesminister davon zu überzeugen, möglichst rasch eine Vereinbarung zu treffen, während von Brentano um Geduld bat. ${ }^{109}$ Shinnar war grob über den Stand der Dinge informiert, doch wichtige Einzelheiten waren ihm offensichtlich entgangen. Die israelische Presse und folglich die Öffentlichkeit wußten noch weniger.

Im September 1955 war eine hochrangige westdeutsche Delegation, angeführt von Adenauer, Hallstein und von Brentano, nach Moskau gereist und hatte sich mit der Sowjetunion auf die Aufnahme diplomatischer Beziehungen geeinigt. Um eine Anerkennungswelle zugunsten der DDR auszuschließen, wurde noch auf dem Rückflug von Moskau ein neuer außenpolitischer Grundsatz ins Leben gerufen, wonach die Bundesrepublik mit Maßnahmen gegen die Staaten drohte, die diplomatische Beziehungen zur DDR aufnehmen sollten. Unterderhand wurde die später nach dem Staatssekretär im Auswärtigen Amt benannte und durch von Brentanos drakonische Anwendung bekannt gewordene „Hallsteindoktrin“, die in ihrer schärfsten Form Staaten mit dem Abbruch der diplomatischen Beziehungen und der Aussetzung von Handelsvorteilen drohte, zu einem teils gewollten, teils ungewollten Hindernis gegen die Normalisierung der Beziehungen zwischen der Bundesrepublik und Israel. 110 Der jüdische Staat war das erste - indirekte Opfer dieser Doktrin und dürfte am längsten unter ihr gelitten haben. Als sich

105 Shinnar an Sharett vom 25. 12. 1955, ISA, 2418/1.

106 SHARETT, Yomanim, Bd. 5, S. 1333.

107 Auszüge aus dem Protokoll der Regierungssitzung vom 16. 1. 1956, ISA, 7229/6a.

108 YEDIOTH ACHRONOTH (Tel Aviv) vom 16.1.1956.

109 Niederschrift über die Besprechung zwischen Shinnar und von Brentano am 28. 1. 1956, ISA, 3309/25.

110 KOSTHORST, Brentano und die deutsche Einheit, S. 88-93; END, Zweimal deutsche Außenpolitik, S. 36-51. 
Jerusalem für Verhandlungen zur Aufnahme von Beziehungen zu Bonn entschied, nahm man dort die neue Realität noch nicht wahr. Vielleicht war es dafür auch noch zu früh.

Im Sommer 1955 führte das Auswärtige Amt bei den Vertretungen der Bundesrepublik im Ausland eine Umfrage über Israel durch. Im Mittelpunkt standen Fragen über die Einstellung der entsprechenden Staaten zu Israel und deren Verhältnis zu den israelischen Vertretungen vor Ort. Zudem wurden die westdeutschen Diplomaten im Ausland um eine Einschätzung der Folgen einer möglichen Aufnahme diplomatischer Beziehungen zwischen der Bundesrepublik und Israel gebeten. ${ }^{111}$ Die meisten Antworten spiegeln freundliche oder neutrale Ansichten sowie Zustimmung zur Normalisierung mit dem jüdischen Staat wider. Eine Ausnahme bildeten die Antworten aus den arabischen Ländern außer Sudan sowie aus einigen islamischen Staaten. Die Berichte westdeutscher Diplomaten im Orient reflektieren die extreme Feindseligkeit ihrer Gastländer gegenüber Israel und in bestimmten Fällen auch eine latente Israelfeindschaft der Verfasser selbst. ${ }^{112}$ Die Diplomaten berichteten über die kategorische Ablehnung jeder Form von Beziehungen zwischen Bonn und Jerusalem und warnten vor harten Gegenmaßnahmen, wie etwa der Aufnahme von diplomatischen Beziehungen zur DDR, dem Abbruch derselben mit der Bundesrepublik, der Aussetzung jeglicher Handels- und Wirtschaftskontakte mit der Bundesrepublik sowie antideutschen Ausschreitungen. Außerdem wurde auf die Gefahr hingewiesen, daß andere westliche Staaten bzw. westliche Firmen versuchen könnten, in die Bresche zu springen, sowie auf den Umstand, daß die Sowjetunion, der Ostblock und der „Weltkommunismus" von einer feindlichen arabischen Reaktion gegen die Bundesrepublik profitieren würden - zum Schaden des Westens. Mehrere westdeutsche Diplomaten äußerten Bedenken zum Luxemburger Abkommen. Bei allen Verständnis für den Hintergrund dieses Abkommens, könne sich die Bundesrepublik keine weitere Annäherung an Israel leisten, schrieb Frhr. von Welck abschließend in seinem Bericht an den Bundesaußenminister vom 28. November 1955. ${ }^{113}$

Die Berichte aus dem Nahen Osten übten großen Einfluß auf die Entscheidungsträger in Bonn aus. Ein Nebeneffekt waren die Spannungen zwischen den Abteilungen 2 und 3 des Auswärtigen Amts. Die Politische Abteilung (Abteilung 2), der unter anderem Blankenhorn und Frowein angehörten, drängte auf eine Annäherung zwischen der Bundesrepublik und Israel. Doch die für den Nahen Osten zuständige Abteilung Länderreferate (Abteilung 3) mit dem „Arabisten“ Frhr. von Welck an der Spitze, an die die arabischen Proteste und die reservierten Berichte westdeutscher Diplomaten in arabischen Ländern gerichtet waren, setzte

111 Runderlaß an alle diplomatischen und konsularischen Vertretungen der Bundesrepublik vom 2. 7. 1955, PA, 308, 210-92/92.19, 1149/55.

112 Botschaft in Amman an das AA vom 18. 7.1955, PA, 316, 81.00, 92.19; von Waldow, Bagdad, an das AA vom 26. 7. 1955 und 2. 8. 1955; die Botschaft in Kairo an das AA vom 5. 8. 1955; die Botschaft in Beirut an das AA vom 23. 8. 1955; Dr. Seydel, Tripolis/Libyen, an das AA vom 28. 10. 1955; die Botschaft in Djidda an das AA vom 29. 11. 1955; Bericht Dr. Melchers nach Bonn vom 15. 8. 1955, PA, 316, 81.00, 92.19.

113 Von Welck an den Außenminister vom 28. 11. 1955, PA, 316, 81.01/1. 
sich durch. Die Israel betreffenden Angelegenheiten wurdem ihrem Aufgabenbereich zugeordnet. ${ }^{114}$

Die inzwischen weiterentwickelte und verfeinerte Hallsteindoktrin, wurde zum Eckpfeiler der westdeutschen Außenpolitik. Sie kam auf mehreren Diplomatenkonferenzen zur Sprache. Die erste dieser Zusammenkünfte im Dezember 1955 befürwortete sie. ${ }^{115}$ Die versammelten hochrangigen Diplomaten warnten vor den Gefahren der Politik der blockfreien Staaten, vor allem Jugoslawiens, Ägyptens und Indiens. Die größte Gefahr für die Bundesrepublik lauerte aber im Nahen Osten. ${ }^{116}$ Noch während der Konferenz wurde sie durch die Ankunft einer DDR-Handelsmission in Kairo am 10. Dezember 1955 veranschaulicht. Die arabischen Staaten, besonders Ägypten und Syrien, gefährdeten den Alleinvertretungsanspruch, worauf sich die Bundesregierung veranlaßt fühlte, ihnen besondere Aufmerksamkeit zu schenken. Vor allem Syrien neigte dazu, diplomatische Beziehungen zur DDR aufzunehmen, und wartete nur noch auf eine günstige Gelegenheit. Die arabischen Drohungen warfen einen dunklen Schatten auf die Beratungen über die Aufnahme diplomatischer Beziehungen zu Israel. Sehr zum Mißfallen des Auswärtigen Amts drohte gerade der Nahe Osten zum ersten Anwendungsgebiet der Hallsteindoktrin zu werden. Man befürchtete ein Junktim, nämlich daß der Austausch diplomatischer Vertretungen mit Israel zu einer Anerkennung der DDR durch die arabischen Staaten führen könnte. Die Verhandlungen über die deutsch-israelischen Beziehungen hatten sich längst zu einem internationalen Problem entwickelt mit Auswirkungen auf weitere Staaten im Nahen Osten und indirekt auch auf die Großmächte und den Kalten Krieg.

Im Herbst 1955 wurde in der Bundesrepublik ein ägyptisch-tschechoslowakisches Waffengeschäft aufgedeckt, das Israel in Angst und Schrecken versetzte. Der jüdische Staat hatte den für die Araber bestimmten Waffen aus dem Ostblock nichts entgegenzusetzen, weder qualitativ noch quantitativ. ${ }^{117}$ In offenen und geschlossenen - beinahe hysterisch geführten - Debatten bekundete die israelische Regierung den Willen, Waffen von jeder sich bietenden Quelle zu kaufen. Verschiedentlich war von der Möglichkeit eines Präventivschlags die Rede, d.h. von einem Militärschlag, bevor die Ostblockwaffen Ägypten erreichen würden und zum Einsatz bereit seien. ${ }^{118}$ Die Krisenstimmung in Israel war von zunehmender politischer Isolation begleitet. Einseitige antiisraelische Beschlüsse des UNOSicherheitsrates, unterstützt vom Ostblock und den Blockfreien, weckten düstere Erinnerungen an den Holocaust, an die Einsamkeit der verfolgten Juden, denen niemand zu Hilfe kam. Das in Israel damals verbreitete Lied mit dem Titel „Die

114 Von Welck an den Außenminister betr. die Israelfrage vom 14. 12. 1955, PA, 316, 81.00/1.

115 KOSTHORST, Brentano und die deutsche Einheit, S. 91-93; END, Zweimal deutsche Außenpolitik, S. 40-41.

116 FRIEDMANN/BüTTNER/HunsLER, Bundesrepublik und arabische Staaten, S. 120-121.

117 Yachil an Eytan o.D. [verm. Ende 1955], ISA, 2539/4.

118 Niederschrift über die Sitzung des Politkomittees von Mapai vom 16. 11. 1955 (vor allem die Reden von Sharett, Aranne und Lavon), BGA, The Protocols File; KNESSET-PROTOKOLLE [Original hebr.], 2. Knesset, 8. Sitzung, S. 87-100, die Reden von Rimalt (Allgemeine Zionisten), Argov (Mapai), Begin (Cherut), Barzilai (Mapam) und die Eröffnungsrede von Ministerpräsident Sharett am 18. 10.1955. 
ganze Welt ist gegen uns" veranschaulicht diese Stimmung. Das Gefühl der Einsamkeit ermunterte die Israelis, sich nach neuen Partnern in Deutschland und anderswo umzusehen. So versuchte die israelische Regierung mit Hilfe der Bundesrepublik freundschaftliche Beziehungen zu postkolonialen Staaten zu knüpfen. Jerusalem war insofern auf Bonns guten Willen angewiesen, als das amerikanische Außenministerium unter Dulles Israel die kalte Schulter zeigte. ${ }^{119}$ Der Generaldirektor des israelischen Außenministeriums, Walter Eytan, appellierte an die westlichen Interessen im Kalten Krieg: Israel sei ein unerschütterlich antikommunistisches Bollwerk im Nahen Osten und verdiene deshalb Unterstützung gegen den sowjetischen Machthunger in der Region. ${ }^{120}$ Das war ein reichlich naives Argument, wenn man die amerikanischen Anstrengungen bedenkt, die arabischen Staaten, besonders Ägypten, in die antikommunistische Front einzureihen. Oder wurde hier versucht, das eigentlich gegen Israel gerichtete Argument der sowjetischen Bedrohung, von dem das Auswärtige Amt demnächst Gebrauch machen sollte, zur Stärkung der israelischen Position zu nutzen? Adenauer und Strauß erschien es schon bald plausibel. Bis es soweit war, wirkte es jedoch zu Israels Ungunsten.

Wie ernst die Lage in Israel empfunden wurde, geht aus den Erörterungen des israelischen Außenministeriums über den Status der Schilumim im Kriegsfall hervor. Die Beratungen konzentrierten sich vor allem auf die rechtlichen, politischen und moralischen Auswirkungen. Die Kölner Mission machte die israelische Regierung darauf aufmerksam, daß die gemischte Kommission zögere, Stahllieferungen zu bewilligen, daß sich der arabische Druck immer stärker bemerkbar mache und daß ein Krieg im Nahen Osten erwartet werde. ${ }^{121}$ In einem Gutachten des juristischen Beirats des israelischen Außenministeriums wurde darauf hingewiesen, daß das Schilumimabkommen keine Sonderbestimmungen für den Kriegsfall enthalte. Auch das internationale Recht biete keine Handhabe für eine solche Situationen, und da die Bundesrepublik nicht Mitglied der UNO sei, seien deren Beschlüsse für Bonn nicht verbindlich. Nur vom Prestige dieser internationalen Organisation her sei ein gewisser Einfluß auf die Bundesregierung zu erwarten. Es müsse, so der Bericht weiter, mit Behinderungen für deutsche Schiffe in dieser Region gerechnet werden. ${ }^{122}$ Eher kurios mutet die Forderung an, der Beamte der Israel-Mission im Kontakt mit deutschen Firmen begegneten: Arabische Vertreter drohten ihnen, daß die Sieger den finanziellen Verpflichtungen Israels im Falle der Vernichtung dieses Staates nicht nachkommen würden, worauf verängstigte deutsche Geschäftspartner von der israelischen Regierung verlangten, Vorkehrungen für diesen Fall zu treffen. ${ }^{123}$

119 Ilsar an Shinnar vom 29. 1. 1956, ISA, 2539/4; Rosenne an Shinnar vom 9. 1. 1956, ISA, 2543/11; Rundbrief des Generaldirektors an israelischen Vertretungen vom 10.1. 1956; Sharett an Shinnar vom 6. 3. 1956; Shinnar an Sharett vom 7. 3. 1955, ISA, 2413/3b; Amiel Najar, Abteilungsleiter Westeuropa, an Sharett und Eytan vom 19. 2. 1956, ISA, 3309/25.

120 Eytan an Shinnar vom 2. 2. 1956, ISA, 3309/25.

121 Shinnar an den Finanzminister vom 6. 2. 1956, ISA, 2542/11.

122 Der Rechtsberater an Shinnar vom 9. 1. 1956, ISA, 2543/11.

123 Dagan an das israelische Außenministerium vom 13. 12. 1955, ISA, 2539/4. 
Jener Teil der westdeutschen Industrie, der in arabische Länder exportierte und dort investierte, sprach sich gegen Beziehungen zu Israel aus und kam damit den Wünschen der arabischen Handelspartner nach. Ähnliche Reaktionen, wenn auch in geringerem Ausmaß, waren bereits vor der Abstimmung über das Schilumimabkommen zu beobachten gewesen. Die westdeutschen Wirtschaftsinteressen im Orient hatten sich inzwischen jedoch stark entwickelt und entsprechend auch ihr Einfluß auf das Auswärtige Amt und das Bundeswirtschaftsministerium. Auch die arabischen Maßnahmen gegen Israel, darunter die Boykottlisten, machten sich immer stärker bemerkbar.

In Erwartung einer Annäherung entschloß sich die israelische Regierung gegenüber der Bundesrepublik zu einer Reihe von administrativen Gesten. Am auffälligsten war die Entfernung des Vermerks "nicht gültig für Deutschland“ aus israelischen Pässen. Der praktische Nutzen dieses Stempels war, wie erwähnt, begrenzt. Eine feindselige Geste, nicht mehr. Zudem hatten die bundesdeutschen Behörden den Vermerk dazu benutzt, um die Einreise von israelischen Staatsbürgern zu beschränken, die sich bereits in großer Zahl in der Bundesrepublik aufhielten. ${ }^{124}$ Die israelische Regierung beschloß weitere Liberalisierungsschritte im gesellschaftlichen Umgang mit deutschen Diplomaten im Ausland, bei akademischen Austauschprogrammen sowie bei der Gewährung von Einreisebewilligungen für westdeutsche Journalisten und andere Besucher aus der Bundesrepublik. ${ }^{125}$ Diese kleinen Schritte sind als eigentlicher Gradmesser der israelischen Haltung gegenüber der Bundesrepublik zu werten. Die Annäherung war stets von solchen Schritten begleitet, die Verschlechterung des gegenseitigen Verhältnisses dagegen von der Annullierung derselben.

Um die Unterstützung der führenden Oppositionspartei, der SPD, für die Aufnahme diplomatischer Beziehungen zu bekommen, hielten Vertreter der IsraelMission deren Organe auf dem laufenden und trafen sich sporadisch mit der Parteiführung. Am 27. Februar 1956 bat Shinnars Stellvertreter, Arthur Bergmann, den SPD-Parteivorsitzenden Erich Ollenhauer bei einem informellen Gespräch um die Unterstützung der SPD für die Aufnahme diplomatischer Beziehungen, die Ollenhauer prompt zusicherte. Der SPD-Parteivorsitzende versprach zudem, mit Brentano zu sprechen und ihn zu einer bedingungslosen Annahme der israelischen Vorschläge aufzufordern. ${ }^{126}$ Zum ersten Mal sah sich Israel gezwungen, bei deutschen Stellen für die Aufnahme diplomatischer Beziehungen zu werben. Man mag sich fragen, weshalb Shinnar seinen Stellvertreter zu Ollenhauer schickte. War es Shinnars persönliche Abneigung gegen die Sozialdemokraten, die ihn von einem Treffen mit dem SPD-Parteivorsitzenden abhielt?

Eine Flut von Gerüchten und widersprüchlichen Erklärungen überschwemmte die Presse und die diplomatischen Akten. Besonders häufig waren Gerüchte über den Beschluß zur Aufnahme diplomatischer Beziehungen zu Israel, wie sie etwa

124 Zeev Scheck an Sharett vom 25. 4. 1956, ISA, 2539/4; Der Leiter der Konsularabteilung im israelischen Außenministerium an den Generaldirektor vom 8. 1. 1956, ISA, 2413/2b; Abteilung Westeurpa an den Generaldirektor vom 15. 1. 1956, ISA, 2539/4.

125 Rundbrief an die israelischen Vertretungen vom 1. 2. 1956, ISA, 3309/25.

126 Shinnar an Sharett vom 29. 2. 1956, ISA, 2526/8. 
die Abteilung 3 im Auswärtigen Amt bewußt verbreitete und später wieder dementierte. Optimistische Äußerungen von Regierungsvertretern und Diplomaten nährten Shinnars vorsichtigen Optimismus und erzeugten falsche Erwartungen in Jerusalem. Am 3. April 1956 berief das Auswärtige Amt in Istanbul eine fünftägige Konferenz von Botschaftern der Bundesrepublik im Orient ein, um über diese Frage zu beraten. Angesichts der Zusammensetzung dieser Konferenz und der schriftlichen Berichte der daran teilnehmenden Botschafter stand das Ergebnis von vornherein fest.

\section{Freunde in der Not ...}

Am 5. Februar 1956 dementierte das Auswärtige Amt eine Mitteilung des SPDPressedienstes, wonach Verhandlungen über die Aufnahme diplomatischer Beziehungen stattfänden. Für die Information war die Israel-Mission verantwortlich. Der rasche Widerruf dieser offenkundig authentischen Nachricht erfolgte aus Angst vor arabischer Vergeltung. ${ }^{127}$ Es war ein Warnsignal für Israel, das zur Verstärkung des israelischen Drucks auf deutsche Persönlichkeiten führte. Goldmann suchte Adenauer auf und rang ihm das Versprechen ab, den Normalisierungsprozeß zu beschleunigen. ${ }^{128}$ Am 6. März 1956 kam es zum entscheidenden Gespräch zwischen von Brentano und Shinnar. Der Bundesaußenminister entschuldigte sich für das Dementi und tat es als Dummheit des Auswärtigen Amts ab. Shinnar faßte das lange und ausführliche Gespräch in zwei Punkten zusammen: Von Brentano wünsche positive Ergebnisse und bitte um Aufschub bis April, dem Zeitpunkt der Botschafterkonferenz. Ein paar Tage später telegraphierte Goldmann Sharett, daß die Bundesrepublik eine Dienststelle in Israel zu errichten wünsche und daß Shinnar demnächst offiziell darüber in Kenntnis gesetzt werde. ${ }^{129}$ Am nächsten Tag, den 14. März, teilte von Brentano Shinnar schriftlich unter anderem mit:

„Mit Beziehung auf unsere Besprechung am 6. d. Mts. teile ich Ihnen mit, daß die Bundesregierung grundsätzlich damit einverstanden ist, in Israel eine Dienststelle zu errichten, die mutatis mutandis - etwa der Israel-Mission entsprechen würde. Der Zeitpunkt der Errichtung dieser Stelle und ihre Aufgaben im einzelnen würden später in Verhandlungen mit Ihnen festzulegen sein. ${ }^{~} 130$

Von Brentano bat, die Angelegenheit streng vertraulich zu behandeln. Shinnar empfing das Schreiben mit großer Genugtuung und wies bei folgenden Besprechungen über die Frage der diplomatischen Beziehungen jeweils auf dessen Inhalt hin. Doch nicht alle Kollegen teilten seine positive Einschätzung. Der deutsche Außenminister hatte nur die Eröffnung einer Mission in Aussicht gestellt, nicht

127 Anlage zu Shinnars Schreiben an Sharett vom 29. 2. 1956 und 2.3. 1956, ISA, 2516/8; MA'ARIV (Tel Aviv) vom 10.2. 1956; Memorandum an den Bundesminister vom 6. 3 . 1956; Aufzeichnung vom 6. 3. 1956, PA, 1024, 708, 82.00-01/70.

128 Telegramm Goldmanns an Sharett vom 13. 2. 1956, CZA, Z 6/1111.

129 Telegramm Goldmanns an Sharett vom 13. 3. 1956, CZA, Z 6/1111.

130 Von Brentano an Shinnar vom 14. 3. 1956, ISA, 3309/13. 
mehr. Zudem schien sein Angebot auf schmalem Fundament zu ruhen. Dennoch verpflichtete es die Bundesrepublik und wurde deshalb auf die Tagesordnung der bevorstehenden Botschafterkonferenz gesetzt.

Auf der Botschafterkonferenz in Istanbul nahmen die Leiter der westdeutschen Gesandtschaften in den arabischen Staaten, in den anliegenden islamischen Staaten (Pakistan, Afghanistan) sowie in Äthiopien teil. Ebenfalls anwesend waren Staatsekretär Hallstein, der die Konferenz leitete, und Vertreter der Abteilungen 2 und 3. Keiner der Anwesenden vertrat die Interessen des Staates Israel mit derselben Fachkenntnis, demselben Verständnis und derselben Aufrichtigkeit, mit denen sich die Diplomaten für die Interessen ihrer jeweiligen arabischen Gastländer einsetzten. ${ }^{131}$ Die Konferenzteilnehmer waren mit den Interessen der Bundesrepublik und der Außenpolitik der Bundesregierung, einschließlich der Hallsteindoktrin, sowie mit den Besonderheiten ihrer Gastländer bestens vertraut. Aber niemand hielt es für nötig, sich näher mit den Bedürfnissen der Israelis und der Stimmung in diesem Land zu befassen. Keiner der anwesenden Diplomaten konnte erklären, weshalb die israelische Regierung beschlossen hatte, einen direkten Kanal zur Bundesrepublik einzurichten. Das Protokoll weist hierzu lapidar auf zwei Möglichkeiten hin: Es sei Sharett gelungen, seine Kollegen von seiner Linie zu überzeugen, und es sei für Israel eine Prestigesache, offizielle Beziehungen mit Bonn aufzunehmen. Kein Wort über die Versuche des jüdischen Staates, die politische Isolierung zu durchbrechen, über die existentielle Notwendigkeit, die Beziehungen zu anderen Ländern zu verbessern, und über die Ängste angesichts der umfangreichen Waffenlieferungen an Ägypten. Die Dokumente zeugen auch von einem akuten Mangel an Übersetzern aus dem Hebräischen.

Die versammelten westdeutschen Diplomaten schätzten ihre Gastländer den Berichten zufolge offensichtlich nicht besonders hoch ein. Die „Orientalen“ seien unstabil, emotional, unfähig zu rationalem Denken und Hitzköpfe. Ihren Forderungen gebe man besser nach, um das Gesicht nicht zu verlieren, hieß es.

Die Botschafterkonferenz diskutierte die Gefahren des israelisch-arabischen Konflikts für die Bundesrepublik und riet zu Neutralität in regionalen Konflikten wie diesem und dem Zypernkonflikt. Besondere Aufmerksamkeit wurde dem Flirt einzelner arabischer Staaten mit dem Sowjetblock und der DDR geschenkt. Hallstein erinnerte an die arabischen Drohungen im Zusammenhang mit der Auseinandersetzung um das Luxemburger Abkommen. Diese, so Hallstein, seien damals ins Leere gelaufen, doch die Lage habe sich seither geändert, und nun dürfe man kein Risiko mehr eingehen. Falls ein arabischer Staat die DDR anerkenne, könnten rasch weitere folgen. Ohne Namen zu nennen, widersprach Hallstein Shinnar und weiteren israelischen Vertretern, die behauptet hatten, daß es sich wie im Frühjahr 1953 auch diesmal um leere Drohungen handle. Die anwesenden Diplomaten warnten vor Illusionen. Die Last des Luxemburger Abkommens sei schwer genug, und Bonn sei gut beraten, sich nicht noch mehr aufzubürden, indem es diplomatische Beziehungen irgendweicher Art zu Israel aufnehme. Man verlange aber nicht, daß die Bundesrepublik ganz auf Beziehungen zu Israel ver-

131 Niederschrift über die Nahostkonferenz in Istanbul am 3.-7.4. 1956, PA, Bd. 162, Sts., $627 / 56$. 
zichte. Es gehe nur darum, den geeigneten Zeitpunkt abwarten, so die Botschafter. Bonn solle zudem der Sowjetunion nicht die Gelegenheit bieten, hieß es weiter, den israelisch-arabischen Konflikt für eigene Belange auszunützen. Wenn es den Russen gelinge, sich im Nahen Osten auszubreiten, würde Israel am meisten darunter leiden. Es sei somit im besten Interesse der Israelis und des Westens, wenn die Bundesrepublik als Teil des Westens vorläufig keine diplomatischen Beziehungen zu Israel aufnehme. Die Bundesrepublik sei frei von belastender imperialistischer und kolonialistischer Vergangenheit und deshalb eher in der Lage, die Interessen des Westens gegenüber den Arabern zu vertreten. Sie sei also gut beraten, ihre eigenen Interessen, etwa im Bereich des Handels und der Investitionen, zu verfolgen und ihren wirtschaftlichen Einfluß auszudehnen, auch gegen den Willen der westlichen Partner. Die lokale Bevölkerung in den arabischen Ländern bewundere die deutsche Gründlichkeit, den deutschen Fleiß, die deutsche Kultur und die deutsche Sprache, wovon die Bundesrepublik und die Bewohner der Region profitieren könnten. Ein verfehlter Annäherungsschritt gegenüber Israel könne all dies und noch mehr gefährden, urteilten die Botschafter.

Es blieb nur die Frage, wie man diese schlechte Nachricht den Israelis beibringen solle. Hallstein schlug vor, eine Verurteilung Israels durch den UNO-Sicherheitsrat abzuwarten. Da damit aber nicht zu rechnen war, einigte man sich schließlich auf den Vorwand der „kommunistischen Gefahr“. Tatsächlich rechtfertigte Hallstein dann Shinnar gegenüber die Entscheidung der Bundesregierung, keine diplomatischen Beziehungen zu Israel aufzunehmen, mit diesem Argument. Das Auswärtige Amt hatte offensichtlich vor den arabischen Drohungen kapituliert, die das ägyptische Staatsoberhaupt, Gamal Abdel Nasser, noch vor Abschluß der Botschafterkonferenz bekräftigt hatte. ${ }^{132}$ Die Furcht vor der Anerkennung der DDR, vor der Ausbreitung des Kommunismus und vor der - in Istanbul nicht erwähnten - Gefährdung der persönlichen Sicherheit deutscher Diplomaten in Israel war zwar aufrichtig, aber nicht entscheidend. Im Vordergrund stand offensichtlich die Werbung um die arabischen Staaten. Der Wunsch, die Beziehungen zwischen der Bundesrepublik und der arabischen Welt zu vertiefen und zu pflegen, wurde deutlich zum Ausdruck gebracht.

Die Debatte über Israel war erstaunlich oberflächlich, und nur wenige Botschafter nahmen aktiv daran teil. Der bundesdeutsche Gesandte in Äthiopien sagte vor den versammelten Botschaftern, Äthiopien würde sich dem Entscheid der Bundesrepublik anschließen, diplomatische Beziehungen zu Israel aufzunehmen. Genau darauf gründeten die Hoffnungen in Jerusalem, nämlich daß Bonn dem jüdischen Staat behilflich sein würde, die Mauer der Ablehnung zu durchbrechen. Doch die Worte des Gesandten in Äthiopien verhallten ungehört. Israel interessierte die Anwesenden nicht im geringsten. Sie waren zusammengekommen, um - verständlicherweise - den Anliegen ihres Landes und nicht denjenigen Israels Gehör zu verschaffen, wenn sie sich auch auf humanitäre Motive beriefen. Die Beschlüsse der Botschafterkonferenz in Istanbul sollten ihre Gültigkeit für zehn Jahre, bis 1965, behalten.

132 Vermerk zur Lage im Nahen Osten, Israelfrage vom 3.-7. 4. 1956, PA, 162, St.Sek. 62756, 3.-7. 4. 56. 
Wie bereits dargelegt, war Israel das erste Opfer der Hallsteindoktrin. Um die arabischen Staaten von Beziehungen zur DDR abzuhalten, bestrafte Bonn Israel, und zwar mit der härtesten Sanktion, die für Verstöße gegen die Doktrin vorgesehen war: mit der Verweigerung der Aufnahme diplomatischer Beziehungen, die dem Abbruch derselben gleichkam. Ähnliche Sanktionen verhängte die Bundesregierung gegen Jugoslawien, Kuba, Tansania, Guinea und andere Staaten, die jedoch im Gegensatz zu Israel aktiv gegen die Interessen der Bundesrepublik verstoßen hatten. Doch die israelische Regierung hatte nichts unternommen, was die Chancen für die Wiedervereinigung Deutschland gefährden könnte.

Die Hallsteindoktrin verwandelte die Bundesrepublik quasi in eine Milchkuh. Die Araber melkten beide deutschen Staaten, Israel nur Westdeutschland. Zur Verdeutlichung ihres Standpunkts erfanden die arabischen Staaten die sogenannte Arefdoktrin, benannt nach dem irakischen Diktator Abdel Rachman Aref (19631964 an der Macht). Dieser drohte der Bundesrepublik mit der Anerkennung der DDR und erzeugte damit ein Junktim. Die Bundesregierung verzichtete auf eine entsprechende Verknüpfung zur Mäßigung der arabischen Haltung, wie etwa die Drohung, Israel anzuerkennen. Gelegentlich machte sie sogar Abstriche beim Alleinvertretungsanspruch. Die wirtschaftliche Zusammenarbeit mit den arabischen Staaten - Handel, Investitionen und Kredite - wurde unter allen Umständen weitergeführt, wenn auch etwa bei Krediten durch die Zinshöhe, die Laufzeit und - in einigen Fällen - auch durch die Umwandlung zu nichtrückzahlbaren Darlehen gewisse Steuerungsmöglichkeiten genutzt wurden. Die Investitionsund Kreditpolitik war ein bewährtes (Schmier-)Mittel zur Erreichung bestimmter politischer Ziele, doch auch die Empfängerstaaten wußten sie zu ihrem Vorteil zu nutzen. Ein deutliches Beispiel war der Bau der Staudämme in Assuan (Ägypten) und am Euphrat (Syrien). Hinzu kamen Waffengeschäfte gegen billige Kredite und manchmal sogar als Geschenk. An solchen Geschäften war auch die Bundesrepublik beteiligt, besonders in den Bereichen Raketen, Flugzeuge sowie der Waffenentwicklung und -produktion in Ägypten. Die „wirtschaftliche Zusammenarbeit" umfaßte Ausbildungs- und Stipendienprogramme, Weiterbildung in der Bundesrepublik auf deutsche Kosten, den Bau von Schulen und Institutionen im kulturellen und technologischen Bereich und sogar Deutschkurse im Empfängerstaat. Der Nutzen der westdeutschen Wirtschaftshilfe für die Empfängerstaaten war erheblich und breit gestreut. Es war den arabischen Staaten offensichtlich gelungen, Bonn nicht nur mehrere politische Kompromisse abzupressen, sondern auch ausgiebig von ihrem guten Willen zu profitieren. Doch die "wirtschaftliche Zusammenarbeit" mit den arabischen Staaten - und mit Israel - war zweifellos auch für die Bundesrepublik mit so manchem nützlichen Nebeneffekt verbunden.

Als Shinnar am 28. Januar 1956 mit von Brentano Vorschläge über die Aufnahme diplomatischer Beziehungen erörterte, unterstrich, wie bereits erwähnt, der Bundesaußenminister die Notwendigkeit, die Angelegenheit zuerst mit den Alliierten zu besprechen. ${ }^{133}$ Die Amerikaner zeigten Verständnis für die besonderen Interessen der Bundesrepublik in der Region: „Die Bundesrepublik Deutsch-

133 Shinnar an Sharett vom 28. 1. 1956, ISA, 3309/25. 
land ist bereit, mit uns im Nahen Osten zusammenzuarbeiten. Andererseits ist davon auszugehen, daß die Bonner Regierung den größten Teil ihrer Energie dafür verwenden wird, die Anerkennung Ostdeutschlands durch die Araber zu verhindern. ${ }^{~}{ }^{134}$ In Anbetracht des amerikanischen Bestrebens, die arabischen Staaten für das antikommunistische Lager zu gewinnen und des amerikanischen Verständnisses für die speziellen Interessen der Bundesrepublik im Nahen Osten, kann man davon ausgehen, daß das amerikanische Außenministerium die westdeutsche Politik gegenüber Israel gebilligt und möglicherweise sogar aktiv unterstützt hat. 135 Man gewinnt verschiedentlich den Eindruck, daß das Auswärtige Amt die angebliche alliierte Intervention als bequemen Vorwand für umstrittene Entscheidungen benutzte. ${ }^{136}$ Ähnliches sollte sich 1963 wiederholen.

Die Botschafterkonferenz in Istanbul empfahl die Ablehnung bzw. den „Aufschub $^{\alpha}$ jeglicher Beziehungen zu Israel. Brentano teilte dies Adenauer mit. Shinnar blieb er eine Antwort schuldig, und mehrere Anzeichen sprechen dafür, daß dies mit Absicht geschah. An Stelle Brentanos fanden sich Staatssekretär Hallstein, der Leiter der politischen Abteilung, Wilhelm Grewe, und Frhr. von Welck zum Treffen mit Shinnar ein, der zuvor vom israelischen Außenministerium Anweisungen für den Fall einer negativen Antwort erhalten hatte. ${ }^{137}$ Das Treffen war unangenehm. Hallstein war zwar gut vorbereitet, aber offensichtlich unfähig, die unangenehme Nachricht in gewünschter Form zu überbringen. Wie vereinbart, erwähnte er die Sowjetunion und den Kommunismus als Grund für die Nichteinhaltung der Zusage von Brentanos vom 14. März und sicherte gleichzeitig zu, sie bei „erster Gelegenheit" doch noch umzusetzen. Der unterwürfige Shinnar riet seinen Vorgesetzten, Hallstein beim Wort zu nehmen. ${ }^{138}$ Sharett, tief gekränkt, lehnte jedoch ab und wies die israelischen Vertretungen im Ausland an, westdeutschen Diplomaten gegenüber Kälte zu zeigen und den Kontakt mit ihnen zu meiden. Der Ministerpräsident legte sein Vorhaben daraufhin endgültig ad acta und trat ein paar Wochen später unter Druck von David Ben Gurion von seinem Amt zurück. Das Debakel in der Deutschlandpolitik hatte ihn offensichtlich entscheidend geschwächt. Beamte im Außenministerium mißtrauten Shinnar und rieten, sich nicht auf ihn zu verlassen. ${ }^{139}$ Der gerade in Jerusalem tagende Zionistenkongreß reagierte mit Protest, doch Goldmann gelang es, eine breite Debatte zum Thema zu verhindern. Der Knessetausschuß für Sicherheit und auswärtige Angelegenheiten empfahl der Regierung, eine Regierungserklärung in der Knesset

134 Staff Study on NSC 5801 vom 16. 1. 1958, Eisenhower Library, White House Office, OSANSA, Box 23, File Policy toward the Near East.

135 Auf den Einfluß der Alliierten auf die westdeutsche Israelpolitik komme ich später zurück. Hier sei nur angemerkt, daß ein Großteil der amerikanischen Dokumente noch unter Verschluß ist.

136 „Es gelang Deutschland, einen Großteil der arabischen Kritik gegen das Reparationsabkommen mit Israel dadurch zum Schweigen zu bringen, daß an geeigneter Stelle jeweils darauf hingewiesen wurde, daß das Abkommen aufgrund von amerikanischem Druck zustande gekommen war": NSC 5727 vom 13. 12. 1957, Dwight D. Eisenhower Library Abilene, Kansas, File Police toward the NE, White House Office, OSANSA, Box 23.

137 Abteilung Westeuropa an Shinnar vom 10. 5. 1956, ISA, 2539/4.

138 Telegramm Shinnars an Sharett vom 15. 5. 1956, ISA, 3309/25.

139 Anonymer Schreiber an Sharett vom 22. 5. 1956, ISA, 2539/4. 
ohne nachfolgende Debatte zu erwägen. ${ }^{140}$ Am 10. Juni 1956 berief Sharett eine Besprechung mit Beratern unter Teilnahme von Goldmann und Shinnar ein. Shinnar äußerte die Ansicht, daß Bonn binnen sechs Monaten Beziehungen zu Israel aufnehmen werde. Man war sich einig, daß sich Israel keinen Konflikt mit Bonn leisten könne. Die Schilumim seien nicht alles, und Israel sei gut beraten, die Beziehungen zur Bundesrepublik auch auf anderen Ebenen zu fördern. Im Endeffekt beschloß die israelische Regierung abzuwarten und die Initiative der Bundesrepublik zu überlassen.

Die SPD hielt sich mit Kritik an der Israelpolitik des Auswärtigen Amts zurück. Sie war weder an der Anerkennung der DDR durch arabische Staaten noch daran interessiert, Israel dafür verantwortlich zu machen. ${ }^{141}$ Die SPD scheute sich nicht, die Bundesregierung bloßzustellen, doch in der Israelfrage zog sie es zu jenem Zeitpunkt vor, ihr die Initiative zu überlassen. Das Motto hieß abwarten. Die Frage der diplomatischen Beziehungen zwischen der Bundesrepublik und Israel war in eine Sackgasse geraten, und niemand wußte, wie es weitergehen sollte. Im Juli 1956 traten die Beziehungen zwischen der Bundesrepublik Deutschland und dem Staat Israel in eine neue Phase.

140 Bericht von Dr. Walter Hirsch vom 5. 6. 1956, PA, 708, 81.00/1-39, 92.19; Schreiben an das Außenministerium betr. Entscheidung des Ausschussses für Sicherheit und Außenpolitik vom 28. 6. 1956, ISA, 2413/3b.

141 Anug an die Abteilung Westeuropa vom 19.6. 1956, ISA, 2539/4. 
